Review Article

\title{
The Efficacy of Guanxinning Injection in Treating Angina Pectoris: Systematic Review and Meta-Analysis of Randomized Controlled Trials
}

\author{
Yongliang Jia, ${ }^{1}$ Siu-wai Leung, ${ }^{1,2}$ Ming-Yuen Lee, ${ }^{1}$ Guozhen Cui, ${ }^{1}$ \\ Xiaohui Huang, ${ }^{1}$ and Fongha Pan ${ }^{1}$ \\ ${ }^{1}$ State Key Laboratory of Quality Research in Chinese Medicine, Institute of Chinese Medical Sciences, University of Macau, \\ Macao SAR, China \\ ${ }^{2}$ School of Informatics, University of Edinburgh, Edinburgh EH8 9AB, UK
}

Correspondence should be addressed to Siu-wai Leung; siu@inf.ed.ac.uk and Ming-Yuen Lee; simonlee@umac.mo

Received 22 July 2012; Revised 20 January 2013; Accepted 21 January 2013

Academic Editor: Myeong Soo Lee

Copyright (C) 2013 Yongliang Jia et al. This is an open access article distributed under the Creative Commons Attribution License, which permits unrestricted use, distribution, and reproduction in any medium, provided the original work is properly cited.

Objective. The randomized controlled trials (RCTs) on Guanxinning injection (GXN) in treating angina pectoris were published only in Chinese and have not been systematically reviewed. This study aims to provide a PRISMA-compliant and internationally accessible systematic review to evaluate the efficacy of GXN in treating angina pectoris. Methods. The RCTs were included according to prespecified eligibility criteria. Meta-analysis was performed to evaluate the symptomatic (SYMPTOMS) and electrocardiographic (ECG) improvements after treatment. Odds ratios (ORs) were used to measure effect sizes. Subgroup analysis, sensitivity analysis, and metaregression were conducted to evaluate the robustness of the results. Results. Sixty-five RCTs published between 2002 and 2012 with 6064 participants were included. Overall ORs comparing GXN with other drugs were 3.32 (95\% CI: $[2.72,4.04])$ in SYMPTOMS and 2.59 (95\% CI: $[2.14,3.15])$ in ECG. Subgroup analysis, sensitivity analysis, and metaregression found no statistically significant dependence of overall ORs upon specific study characteristics. Conclusion. This meta-analysis of eligible RCTs provides evidence that GXN is effective in treating angina pectoris. This evidence warrants further RCTs of higher quality, longer follow-up periods, larger sample sizes, and multicentres/multicountries for more extensive subgroup, sensitivity, and metaregression analyses.

\section{Introduction}

Ischemic heart disease (IHD) is a major cause of death and global healthcare burden [1]. Angina pectoris, a symptom of IHD, is a severe chest pain due to ischemia of the heart muscle, during obstruction or spasm of the coronary arteries [2]. In the United States, IHD accounts for $26.6 \%$ of all deaths in 2005 , with an age-adjusted male-to-female mortality ratio of 1.5 [3]. The morbidity and mortality of angina in middleaged and elderly people were ranked the top among all common diseases in China [4]. Three categories of conventional Western medicine including nitrates (e.g., isosorbide mononitrate), beta-receptor blockers (e.g., atenolol), and calcium channel blockers (e.g., amlodipine) are commonly used in treating angina [3].
Guanxinning injection (GXN, also known as Danshen Chuanxiong Injection) comprises extracts from two wellknown traditional Chinese medicines Danshen (Salvia miltiorrhiza) and Chuanxiong (Ligustrazine, Ligustium Wallichii Franch) [5]. Danshen and its active compounds tanshinones and isotanshinones have bioactivities against myocardial ischemia, inflammation, and angiotensin-converting enzyme [6]. Chuanxiong and its active compounds tetramethylpyrazine and ferulic acid can dilate coronary arteries, increase myocardial oxygen, and decrease platelet aggregation and thrombosis [7].

GXN was tested to be more effective than nitrates [8], beta-receptor blockers [9], and calcium channel blockers [10] in treating angina. Since the launch of GXN (2002) and prior to this study, there has been only one systematic review, 
which is not compliant with PRISMA [11] and includes only nine randomized controlled trials (RCTs) published in Chinese between 2002 and 2010 [12]. The methods and results of quality assessment of the included RCTs were not clearly reported in the systematic review. Sensitivity and subgroup analyses were missing. Hence, this study aims to provide an internationally accessible, comprehensive, and timely systematic review and meta-analysis in compliance with PRISMA to assess the efficacy of GXN as a monotherapy and combined therapy with conventional Western or Chinese medicines in treating angina pectoris.

\section{Methods}

The procedures of this systematic review and meta-analysis were conducted in accordance with the PRISMA guideline [11], including the search and selection of studies, data extraction from the studies, and meta-analysis (overall, subgroup, sensitivity, publication bias, and metaregression analysis).

2.1. Search Strategies. RCTs published on the efficacy of GXN in treating angina pectoris were retrieved from major bibliographical databases including Medline, PubMed, Cochrane Library, ScienceDirect, Embase, China National Knowledge Infrastructure (CNKI), WanFang Data, China Master Theses Full-text Database (CMTD), and China Doctor Dissertations Full-text Database (CDMD) between the inception dates of databases and 2012 (last search on 18 March 2012). A simple search strategy, that is, searching for the keywords "Guanxinning" or "danshen chuanxiong" or "danshenchuanxiong," was used to search all fields. For instance, the search in WanFang Data using the keyword "Guanxinning" found 196 records and "danshen chuanxiong" found 17 records and "danshenchuanxiong" found none. Exact search strategies and query syntax for specific databases were customized according to the same strategy.

2.2. Study Selection. Inclusion criteria for each study were (a) the participants were suffering from and being treated for angina pectoris; (b) the study was claimed as an RCT; (c) the study compared the efficacy of GXN with conventional (Western and Chinese medicine) drugs. Exclusion criteria were (a) the study was a duplicated or redundant publication and (b) the study did not include symptomatic improvement as a major outcome.

Two reviewers (Y. Jia and F. Pan) independently searched the databases and selected studies according to the inclusion and exclusion criteria. Disagreements between reviewers were resolved by consensus after discussion. Figure 1 shows a flow diagram of study selection.

2.3. Data Extraction. Two reviewers (Y. Jia and F. Pan) independently extracted data items, including (a) years of publication; (b) numbers of authors; (c) follow-up periods; (d) baseline characteristics of participants between groups; (e) sample sizes; (f) outcome measures; (g) dosages and follow-up periods; (h) type of angina; (i) frequencies of adverse events (AE); and (j) the type of angina.

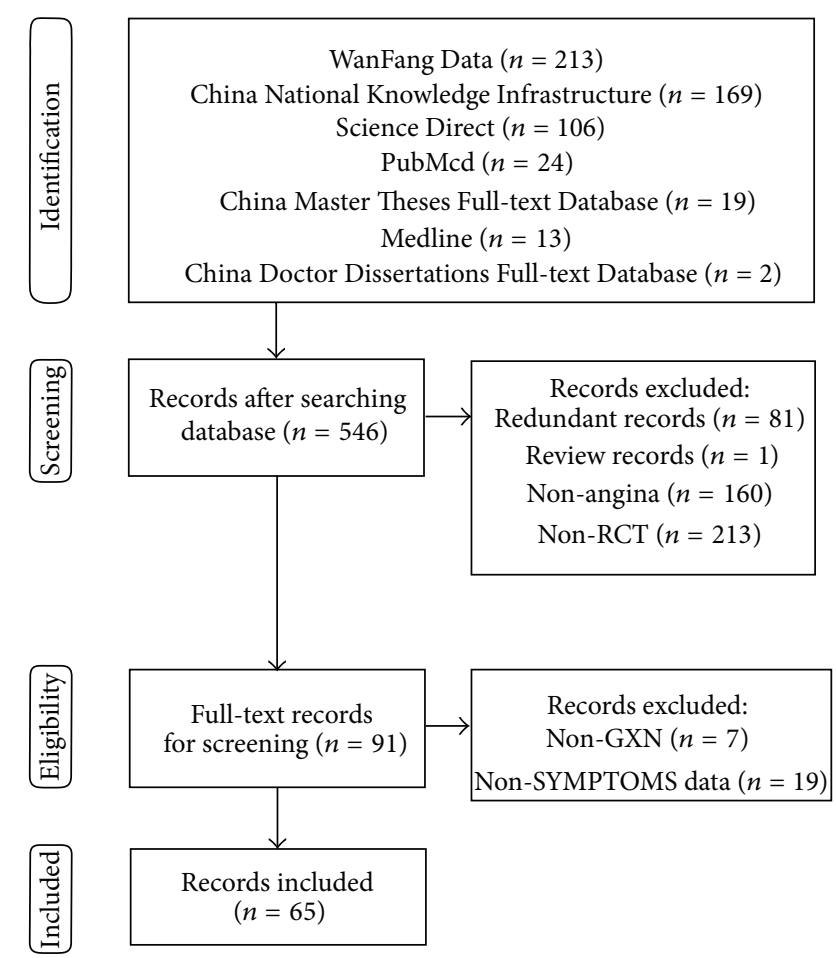

FIgURE 1: Process of searching and screening studies.

2.4. Quality Assessment of Included Studies. Two reviewers (Y. Jia and F. Pan) independently assessed the quality of the included studies according to the Jadad scale [13], its refined version the $M$ scale [14], and the Cochrane Collaboration's tool for assessing risk of bias [15]. The Jadad scale focused on three criteria including "randomization," "blinding," and "dropouts" for assessing the quality of RCT. The $M$ scale added two criteria "baseline comparison of participants" and "adverse event report" on top of the Jadad scale. The Cochrane Collaboration's tool for assessing risk of bias includes "random sequence generation," "allocation concealment," "blinding of participants and personnel," "blinding of outcome assessment (patient-reported outcomes)," "blinding of outcome assessment (SYMPTOMS)," "incomplete outcome data addressed," "reporting bias," and "other sources of bias."

2.5. Criteria for Symptomatic and ECG Improvements. Effective symptomatic improvements should achieve at least $50 \%$ (basic) or $80 \%$ (significant) reduction in frequency of feeling angina chest pain [16]. Effective ECG improvements should achieve (a) at least $0.05 \mathrm{mV}$ lowering at ST segment in ECG (basic) or (b) nearly normal (significant) ECG during an exercise test according to the International Society and Federation of Cardiology/World Health Organization [16].

2.6. Meta-Analysis. Effect sizes were represented by odds ratios (ORs) [17] and their 95\% confidence intervals (CI) [18]. Overall meta-analysis and subgroup analysis employed 
the random-effects model for conservative generalizability. Heterogeneity among studies was assessed by Chi-squared $\left(\chi^{2}\right)$ and I-squared $\left(I^{2}\right)$ tests [19].

2.7. Subgroup and Sensitivity Analyses. Subgroup analysis was conducted to evaluate the overall effects in the subgroups according to years of publication $(\leq 2008$ or $>2008)$, numbers of authors ( 1 or $>1$ ), follow-up periods ( $\leq 14$ days or $>14$ days), sample sizes $(<$ mean sample size or $\geq$ mean sample size), quality scores of the studies ( $<$ mean or $\geq$ mean), different type of angina, and different daily dosage of GXN. The overall effects were also analyzed in subgroups of GXN for monotherapy and adjunctive therapy. Sensitivity analysis was carried out according to different criteria outcomes (basic or significant) in SYMPTOMS and ECG and excluding studies with maximum GXN dosage to assess their influence on the overall effect sizes. The Mann-Whitney-Wilcoxon test was used to compare two subgroups. The Kruskal-Wallis test and the Bonferroni correction were used to compare multiple subgroups. Kendall correlation between ORs of symptoms and ECG was performed.

2.8. Metaregression and Risk of Bias across Studies. Funnel plots [20], Begg's test [21], and Egger's test [22] were employed to assess publication bias. Trim-and-fill method [23] was conducted to identify and correct the funnel plot asymmetry arising from publication bias. Metaregression [24] was conducted to find the possible relationship between the overall effects and the factors such as sample sizes, follow-up periods, $M$ scores, and years of publication.

2.9. Adverse Events. Information about adverse events (AEs) of RCTs, including nonreported adverse events and types and frequency of adverse events reported, was tabulated and analyzed by basic statistics.

2.10. Statistical Analysis. All data analyses, including metaanalysis, forest plot generation, funnel plot generation, metaregression, Kendall correlation, Mann-WhitneyWilcoxon test, Kruskal-Wallis test, Begg's test, and Egger's test, were performed using statistical software R [25] and its "metafor" package for meta-analysis. $P$ values lower than 0.05 were considered statistically significant.

\section{Results}

3.1. Study Selection. Figure 1 depicts the process of study selection. The search of bibliographical databases found 401 records, including 196 records from WanFang Data, 162 records from CNKI, 19 records from CMTD, 11 records from ScienceDirect, 6 records from Medline, 5 records from PubMed, and 2 records from CDMD. According to prespecified selection criteria as described in Methods, 65 studies [26-90] were included for further quality assessment and meta-analysis.

3.2. Study Characteristics. Table 1 lists the main characteristics of the included studies. All included studies were published in the Chinese language between 2004 and 2011 with a total of 6064 participants. The mean sample size was 93.3 (median: 88.0; 95\% CI: [56.5, 130.1]). The follow-up periods were between 1 and 30 days. GXN was compared with the conventional treatments in the included RCTs. Drugs in control group mainly included nitrates, beta-receptor blockers, calcium channel blockers, angiotensin-converting enzyme inhibitors, and some conventional Chinese medicinal products for treating heart disease. Fifty-nine out of 65 RCTs employed GXN plus the conventional treatments in the treatment group while the conventional treatments were employed in control group. Dosage details were listed in Supplementary Table 1 in the Supplementary Material available online at http://dx.doi.org/10.1155/2013/282707. For outcome measures, all 65 included studies reported symptomatic (SYMPTOMS) changes while 38 studies also reported ECG changes.

3.3. Quality Assessment of Included Studies. Table 2 shows the results of quality assessment according to the Jadad scales, $M$ scales, and the Cochrane Collaboration's tool. According to the Jadad scale (with a possible range between 0 and 5 points), 63 studies of all included studies scored 2 with two items "randomization" and "dropouts," one study [34] scored 3, and one study [47] scored 4 . According to the $M$ scale, six studies scored 2, three studies scored 2.5, 30 studies scored 3, 24 studies scored 4 , and 2 studies scored 5 . Fifty included studies reported baseline comparison of participants in experiment and control groups. Thirty-one studies did not report adverse events. Three studies reported types of adverse events. Thirtyone studies reported types and numbers of adverse events. The assessment results of the Cochrane Collaboration's tool showed (1) low risk of bias in random sequence generation for selection bias, blinding of outcome assessment (SYMPTOMS) for detection bias, and incomplete outcome data addressed for attrition bias, (2) high risk of bias in allocation concealment for selection bias, blinding of participants and personnel for performance bias, blinding of outcome assessment (patient-reported outcomes) for detection bias, and reporting bias for selecting reporting, and (3) unclear risk of bias in other sources of bias for other bias.

3.4. Overall Effects of Included Studies. As shown in Figure 2 and Table 3, the overall OR of SYMPTOMS was 3.32 (95\% CI: $[2.72,4.04], Z=11.93, P<0.0001)$ with significant heterogeneity (tau $\left.=0.23, I^{2}=37 \%, P=0.0030\right)$ among the 65 studies with SYMPTOMS outcome. Figure 3 and Table 4 show that the overall OR of ECG was 2.59 (95\% CI: [2.14, 3.15], $Z=9.68, P<0.0001$ ) with nonsignificant heterogeneity (tau $\left.=0.11, I^{2}=32 \%, P=0.0539\right)$ among the 38 studies with ECG outcome. Both ORs (SYMPTOMS and ECG) indicated that GXN was more effective than the drugs in control group in treating angina pectoris. The Kendall correlation between SYMPTOMS and ECG in ORs was statistically significant $($ tau $=0.2644 ; P=0.0200)$.

3.5. Subgroup Analysis. ORs of the subgroups in both SYMPTOMS (Table 3) and ECG (Table 4) were compared based 
TABLE 1: Characteristics of the included studies.

\begin{tabular}{|c|c|c|c|c|c|c|c|c|c|}
\hline Study & $\begin{array}{c}\text { Number } \\
\text { of } \\
\text { authors }\end{array}$ & $\begin{array}{l}\text { Trial date } \\
\text { report }\end{array}$ & $\begin{array}{l}\text { Sample } \\
\text { size }\end{array}$ & $\begin{array}{l}\text { Followup } \\
\text { (day) }\end{array}$ & $\begin{array}{c}\text { Baseline } \\
\text { comparison }\end{array}$ & $\mathrm{AE}$ & $\begin{array}{l}\text { Outcomes } \\
\text { measure }\end{array}$ & Treatment group dosage & Angina \\
\hline Chen 2009 & 1 & 1 & 100 & 15 & 1 & 0 & SYM, ECG & GXN $20 \mathrm{~mL} / \mathrm{d}+\mathrm{CG}$ & Angina \\
\hline $\begin{array}{l}\text { Chen et al. } \\
2011\end{array}$ & 3 & 1 & 100 & 10 & 0 & 0 & SYM & GXN $20 \mathrm{~mL} / \mathrm{d}+\mathrm{CG}$ & Angina \\
\hline Chen 2006 & 1 & 1 & 62 & 14 & 1 & 1 & SYM, ECG & GXN $20 \mathrm{~mL} / \mathrm{d}+\mathrm{CG}$ & Angina \\
\hline $\begin{array}{l}\text { Cheng and } \\
\text { Zang } 2010\end{array}$ & 2 & 1 & 43 & 14 & 1 & 0 & SYM & $\mathrm{GXN} 30 \mathrm{~mL} / \mathrm{d}+\mathrm{CG}$ & Unstable \\
\hline $\begin{array}{l}\text { Cheng et } \\
\text { al. } 2011\end{array}$ & 3 & 1 & 76 & 14 & 1 & 1 & SYM, ECG & GXN $30 \mathrm{~mL} / \mathrm{d}+\mathrm{CG}$ & Angina \\
\hline $\begin{array}{l}\text { Dong XP } \\
2009\end{array}$ & 1 & 0 & 100 & 1 & 0 & 0.5 & SYM & GXN $20 \mathrm{~mL} / \mathrm{d}$ & Angina \\
\hline $\begin{array}{l}\text { Fu and } \\
\text { Meng } 2011\end{array}$ & 2 & 0 & 47 & 10 & 1 & 0 & SYM, ECG & GXN $20 \mathrm{~mL} / \mathrm{d}+\mathrm{CG}$ & Angina \\
\hline $\begin{array}{l}\text { Fu et al. } \\
2010\end{array}$ & 4 & 1 & 56 & 14 & 1 & 0 & SYM, ECG & $\begin{array}{c}\text { GXN } 200 \mathrm{ml}+\mathrm{CG}+\text { shenmaiyin } \\
40 \mathrm{ml}\end{array}$ & Angina \\
\hline $\begin{array}{l}\text { Gao et al. } \\
2005\end{array}$ & 3 & 1 & 60 & 14 & 1 & 1 & SYM, ECG & GXN 20 mL/d & Angina \\
\hline $\begin{array}{l}\text { Gong et al. } \\
2009\end{array}$ & 3 & 1 & 85 & 14 & 1 & 1 & SYM, ECG & $\begin{array}{c}\text { GXN } 20 \mathrm{~mL} / \mathrm{d}+\text { xueshuantong } \\
20 \mathrm{ml}\end{array}$ & Stable \\
\hline $\begin{array}{l}\text { He and } \\
\text { Meng } 2007\end{array}$ & 1 & 1 & 49 & 15 & 1 & 0 & SYM & $\mathrm{GXN} 20 \mathrm{~mL} / \mathrm{d}+\mathrm{CG}$ & Unstable \\
\hline He 2009 & 1 & 1 & 120 & 28 & 1 & 1 & SYM, ECG & GXN $30 \mathrm{~mL} / \mathrm{d}+$ atorvastatin $10 \mathrm{mg}$ & Unstable \\
\hline $\begin{array}{l}\text { Hou and } \\
\text { Gao } 2009\end{array}$ & 2 & 1 & 128 & 14 & 1 & 1 & SYM, ECG & $\mathrm{GXN} 20 \mathrm{~mL} / \mathrm{d}+\mathrm{CG}$ & Stable \\
\hline $\begin{array}{l}\text { Huang et } \\
\text { al. } 2011\end{array}$ & 4 & 0 & 120 & 7 & 1 & 1 & SYM & $\begin{array}{l}\text { GXN } 20 \mathrm{~mL} / \mathrm{d}+\mathrm{CG}+ \\
\text { xueshuantong } 400 \mathrm{mg}\end{array}$ & Angina \\
\hline $\begin{array}{l}\text { Jiang et al. } \\
2010\end{array}$ & 3 & 1 & 116 & 10 & 1 & 0 & SYM, ECG & GXN $20 \mathrm{~mL} / \mathrm{d}+\mathrm{CG}$ & Unstable \\
\hline Jiang 2009 & 1 & 1 & 68 & 20 & 1 & 1 & SYM, ECG & $\mathrm{GXN} 20 \mathrm{~mL} / \mathrm{d}$ & Angina \\
\hline $\begin{array}{l}\text { Jiang et al. } \\
2010\end{array}$ & 5 & 0 & 56 & 7 & 0 & 1 & SYM & GXN $30 \mathrm{~mL} / \mathrm{d}$ & Angina \\
\hline Kong 2009 & 1 & 0 & 100 & 14 & 1 & 1 & SYM & $\mathrm{GXN} 30 \mathrm{~mL} / \mathrm{d}+\mathrm{CG}$ & Unstable \\
\hline $\begin{array}{l}\text { Lan et al. } \\
2006\end{array}$ & 3 & 1 & 64 & 14 & 1 & 1 & SYM & GXN 20 mL/d & Angina \\
\hline $\begin{array}{l}\mathrm{Li} \text { and Jia } \\
2011\end{array}$ & 2 & 1 & 200 & 14 & 1 & 0 & SYM, ECG & $\mathrm{GXN} 30 \mathrm{~mL} / \mathrm{d}+\mathrm{CG}$ & Angina \\
\hline $\begin{array}{l}\text { Li and Lei } \\
2005\end{array}$ & 2 & 1 & 156 & 14 & 0 & 1 & SYM, ECG & $\mathrm{GXN} 20 \mathrm{~mL} / \mathrm{d}+\mathrm{CG}$ & Angina \\
\hline $\begin{array}{l}\text { Li et al. } \\
2009\end{array}$ & 5 & 1 & 168 & 14 & 1 & 0 & SYM & $\mathrm{GXN} 20 \mathrm{~mL} / \mathrm{d}+\mathrm{CG}$ & Angina \\
\hline $\begin{array}{l}\text { Li and Ran } \\
2009\end{array}$ & 2 & 1 & 160 & 10 & 1 & 1 & SYM & $\mathrm{GXN} 20 \mathrm{~mL} / \mathrm{d}+\mathrm{CG}$ & Angina \\
\hline Li 2004 & 1 & 0 & 83 & 7 & 1 & 0 & SYM, ECG & GXN $20 \mathrm{~mL} / \mathrm{d}+\mathrm{CG}$ & Unstable \\
\hline $\begin{array}{l}\text { Liang and } \\
\text { Feng } 2010\end{array}$ & 2 & 0 & 120 & 14 & 1 & 0 & SYM, ECG & GXN $20 \mathrm{~mL} / \mathrm{d}+\mathrm{CG}$ & Unstable \\
\hline Liu 2004 & 1 & 1 & 104 & 10 & 1 & 1 & SYM & GXN $20 \mathrm{~mL} / \mathrm{d}+\mathrm{CG}$ & Unstable \\
\hline $\begin{array}{l}\text { Liu and } \mathrm{Li} \\
2007\end{array}$ & 2 & 1 & 88 & 12 & 0 & 1 & SYM & GXN $20 \mathrm{~mL} / \mathrm{d}+\mathrm{CG}$ & Unstable \\
\hline Liu 2005 & 1 & 1 & 80 & 30 & 1 & 0 & SYM, ECG & GXN $20 \mathrm{~mL} / \mathrm{d}+\mathrm{CG}$ & Unstable \\
\hline Liu 2011 & 1 & 1 & 152 & 28 & 1 & 0 & SYM, ECG & $\mathrm{GXN} 20 \mathrm{~mL} / \mathrm{d}+\mathrm{CG}$ & Angina \\
\hline
\end{tabular}


TABLE 1: Continued.

\begin{tabular}{|c|c|c|c|c|c|c|c|c|c|}
\hline Study & $\begin{array}{c}\text { Number } \\
\text { of } \\
\text { authors }\end{array}$ & $\begin{array}{l}\text { Trial date } \\
\text { report }\end{array}$ & $\begin{array}{l}\text { Sample } \\
\text { size }\end{array}$ & $\begin{array}{l}\text { Followup } \\
\text { (day) }\end{array}$ & $\begin{array}{c}\text { Baseline } \\
\text { comparison }\end{array}$ & $\mathrm{AE}$ & $\begin{array}{l}\text { Outcomes } \\
\text { measure }\end{array}$ & Treatment group dosage & Angina \\
\hline $\begin{array}{l}\text { Lu et al. } \\
2006\end{array}$ & 3 & 1 & 68 & 30 & 1 & 0 & SYM & GXN $30 \mathrm{~mL} / \mathrm{d}+\mathrm{CG}$ & Angina \\
\hline $\begin{array}{l}\text { Ma and } \\
\text { Peng } 2008\end{array}$ & 2 & 1 & 120 & 14 & 1 & 0 & SYM & GXN $30 \mathrm{~mL} / \mathrm{d}+\mathrm{CG}$ & Unstable \\
\hline $\begin{array}{l}\text { Nie and } \\
\text { Chen } 2007\end{array}$ & 2 & 1 & 60 & 14 & 1 & 0 & SYM, ECG & GXN $20 \mathrm{~mL} / \mathrm{d}+\mathrm{CG}$ & Angina \\
\hline $\begin{array}{l}\text { Qiao and } \\
\text { Wu } 2004\end{array}$ & 2 & 0 & 81 & 28 & 1 & 1 & SYM, ECG & GXN $20 \mathrm{~mL} / \mathrm{d}+\mathrm{CG}$ & Stable \\
\hline Song 2010 & 1 & 1 & 82 & 7 & 0 & 0.5 & SYM & $\begin{array}{c}\mathrm{GXN} 20 \mathrm{~mL} / \mathrm{d}+\mathrm{CG}+\text { diltiazem } \\
90 \mathrm{mg} / \mathrm{d}\end{array}$ & Unstable \\
\hline Su 2009 & 1 & 1 & 90 & 15 & 0 & 1 & SYM, ECG & GXN $6 \mathrm{~mL} / \mathrm{d}+\mathrm{CG}$ & Angina \\
\hline Sun 2010 & 1 & 1 & 90 & 14 & 1 & 0 & SYM, ECG & GXN $30 \mathrm{~mL} / \mathrm{d}+\mathrm{CG}$ & Unstable \\
\hline $\begin{array}{l}\text { Sun et al. } \\
2006\end{array}$ & 5 & 1 & 98 & 15 & 0 & 0 & SYM & GXN $20 \mathrm{~mL} / \mathrm{d}+\mathrm{CG}$ & Angina \\
\hline $\begin{array}{l}\text { Tian and } \\
\text { Wu } 2006\end{array}$ & 2 & 1 & 62 & 14 & 1 & 1 & SYM & GXN $30 \mathrm{~mL} / \mathrm{d}+\mathrm{CG}$ & Unstable \\
\hline $\begin{array}{l}\text { Wan and } \\
\text { Xu } 2009\end{array}$ & 2 & 0 & 120 & 14 & 1 & 1 & SYM, ECG & GXN $30 \mathrm{~mL} / \mathrm{d}+\mathrm{CG}$ & Unstable \\
\hline Wang 2007 & 1 & 1 & 100 & 14 & 1 & 1 & SYM, ECG & GXN $30 \mathrm{~mL} / \mathrm{d}+\mathrm{CG}$ & Angina \\
\hline Wang 2011 & 1 & 1 & 85 & 14 & 1 & 1 & SYM, ECG & GXN $20 \mathrm{~mL} / \mathrm{d}+\mathrm{CG}$ & Unstable \\
\hline Wang 2011 & 2 & 1 & 112 & 14 & 1 & 1 & SYM, ECG & GXN $20 \mathrm{~mL} / \mathrm{d}+\mathrm{CG}$ & Unstable \\
\hline $\begin{array}{l}\text { Wang and } \\
\text { Ji } 2008\end{array}$ & 2 & 1 & 60 & 14 & 0 & 0 & SYM, ECG & GXN $20 \mathrm{~mL} / \mathrm{d}+\mathrm{CG}$ & Unstable \\
\hline $\begin{array}{l}\text { Wang and } \\
\text { Sun } 2007\end{array}$ & 2 & 1 & 92 & 10 & 0 & 0 & SYM & GXN $20 \mathrm{~mL} / \mathrm{d}+\mathrm{CG}$ & Unstable \\
\hline Wang 2005 & 2 & 1 & 60 & 15 & 1 & 0 & SYM & GXN $20 \mathrm{~mL} / \mathrm{d}+\mathrm{CG}$ & Unstable \\
\hline Wang 2010 & 1 & 0 & 80 & 14 & 1 & 1 & SYM, ECG & GXN $20 \mathrm{~mL} / \mathrm{d}+\mathrm{CG}$ & Stable \\
\hline Wang 2005 & 1 & 0 & 76 & 15 & 0 & 0 & SYM & $\begin{array}{l}\text { GXN } 20 \mathrm{~mL} / \mathrm{d}+\mathrm{CG}+\text { shenmai } \\
30 \mathrm{~mL} / \mathrm{d}+\text { tongxinluo } 9 \text { pills } / \mathrm{d}\end{array}$ & Unstable \\
\hline Wang 2005 & 1 & 1 & 60 & 14 & 1 & 1 & SYM & GXN $20 \mathrm{~mL} / \mathrm{d}+\mathrm{CG}$ & Unstable \\
\hline $\begin{array}{l}\text { Wang et al. } \\
2011\end{array}$ & 4 & 1 & 60 & 14 & 1 & 1 & SYM & GXN $20 \mathrm{~mL} / \mathrm{d}+\mathrm{CG}$ & Unstable \\
\hline $\begin{array}{l}\text { Wu et al. } \\
2008\end{array}$ & 3 & 0 & 108 & 14 & 1 & 0 & SYM & GXN $20 \mathrm{~mL} / \mathrm{d}+\mathrm{CG}$ & Angina \\
\hline $\begin{array}{l}\text { Wu et al. } \\
2011\end{array}$ & 4 & 1 & 144 & 7 & 1 & 0 & SYM & $\begin{array}{c}\text { GXN } 20 \mathrm{~mL} / \mathrm{d}+\mathrm{CG}+\text { shenmai } \\
50 \mathrm{~mL} / \mathrm{d}\end{array}$ & Unstable \\
\hline Xia 2011 & 1 & 1 & 90 & 14 & 1 & 0 & SYM, ECG & GXN $30 \mathrm{~mL} / \mathrm{d}+\mathrm{CG}$ & Unstable \\
\hline $\begin{array}{l}\text { Yang and } \\
\text { Ma } 2008\end{array}$ & 2 & 1 & 90 & 14 & 1 & 0 & SYM, ECG & $\mathrm{GXN} 30 \mathrm{~mL} / \mathrm{d}+\mathrm{CG}$ & Unstable \\
\hline $\begin{array}{l}\text { Ye et al. } \\
2008\end{array}$ & 3 & 0 & 76 & 15 & 0 & 1 & SYM & GXN $20 \mathrm{~mL} / \mathrm{d}+\mathrm{CG}$ & Unstable \\
\hline $\begin{array}{l}\text { Yu and } \\
\text { Wang } \\
2009\end{array}$ & 2 & 1 & 75 & 15 & 1 & 0 & SYM, ECG & GXN $20 \mathrm{~mL} / \mathrm{d}+\mathrm{CG}$ & Angina \\
\hline Yuan 2005 & 1 & 0 & 104 & 14 & 1 & 0 & SYM, ECG & GXN $20 \mathrm{~mL} / \mathrm{d}+\mathrm{CG}$ & Angina \\
\hline $\begin{array}{l}\text { Zhang } \\
2005\end{array}$ & 1 & 1 & 60 & 14 & 1 & 0 & SYM, ECG & GXN $10 \mathrm{~mL} / \mathrm{d}$ & Angina \\
\hline $\begin{array}{l}\text { Zhang } \\
2010\end{array}$ & 1 & 1 & 240 & 15 & 1 & 1 & SYM, ECG & GXN $20 \mathrm{~mL} / \mathrm{d}+\mathrm{CG}$ & Unstable \\
\hline $\begin{array}{l}\text { Zhang } \\
2004\end{array}$ & 1 & 1 & 102 & 14 & 1 & 1 & SYM, ECG & $\begin{array}{c}\text { GXN } 10 \mathrm{~mL} / \mathrm{d}+\mathrm{CG}+\text { ginkgo leaf } \\
\text { injection } 10 \mathrm{~mL} / \mathrm{d}\end{array}$ & Angina \\
\hline
\end{tabular}


TABle 1: Continued.

\begin{tabular}{|c|c|c|c|c|c|c|c|c|c|}
\hline Study & $\begin{array}{c}\text { Number } \\
\text { of } \\
\text { authors }\end{array}$ & $\begin{array}{l}\text { Trial date } \\
\text { report }\end{array}$ & $\begin{array}{l}\text { Sample } \\
\text { size }\end{array}$ & $\begin{array}{l}\text { Followup } \\
\text { (day) }\end{array}$ & $\begin{array}{l}\text { Baseline } \\
\text { comparison }\end{array}$ & $\mathrm{AE}$ & $\begin{array}{l}\text { Outcomes } \\
\text { measure }\end{array}$ & Treatment group dosage & Angina \\
\hline $\begin{array}{l}\text { Zhang } \\
2004\end{array}$ & 1 & 1 & 42 & 7 & 0 & 1 & SYM & GXN $20 \mathrm{~mL} / \mathrm{d}+\mathrm{CG}$ & Angina \\
\hline $\begin{array}{l}\text { Zhao et al. } \\
2010\end{array}$ & 6 & 1 & 100 & 14 & 1 & 1 & SYM, ECG & $\begin{array}{l}\text { GXN } 10 \mathrm{~mL} / \mathrm{d}+\mathrm{CG}+ \\
\text { xueshuangtong } 120 \mathrm{mg}\end{array}$ & Unstable \\
\hline $\begin{array}{l}\text { Zhao and } \\
\text { An } 2008\end{array}$ & 2 & 1 & 90 & 28 & 1 & 1 & SYM, ECG & $\begin{array}{c}\mathrm{GXN} 20 \mathrm{~mL} / \mathrm{d}+\mathrm{CG}+\text { simvastatin } \\
10-20 \mathrm{mg} / \mathrm{d}\end{array}$ & Unstable \\
\hline Zhao 2010 & 1 & 1 & 86 & 14 & 1 & 0 & SYM, ECG & GXN $30 \mathrm{~mL} / \mathrm{d}+\mathrm{CG}$ & Angina \\
\hline $\begin{array}{l}\text { Zhong et } \\
\text { al. } 2007\end{array}$ & 8 & 1 & 60 & 10 & 0 & 0 & SYM & GXN $20 \mathrm{~mL} / \mathrm{d}+\mathrm{CG}$ & Angina \\
\hline Zhu 2005 & 1 & 1 & 80 & 15 & 0 & 0.5 & SYM, ECG & GXN $20 \mathrm{~mL} / \mathrm{d}+\mathrm{CG}$ & Unstable \\
\hline
\end{tabular}

GXN is Guanxinning injection; LMWH is low molecular weight heparin; and shenmai is Shenmai injection. CG is interventions of control group; SYM is SYMPTOMS; ECG is electrocardiogram; and AE is adverse event. The column of "Trial date report" shows that study did (1) or did not (0) report the trial date. The column of "Baseline comparison" shows that the study did (1) or did not (0) report the baseline comparison between the treatment and control groups.

on the study characteristics including $M$ scores ( $\leq 3$ or $>3$ ), sample sizes ( $<93$ or $\geq 93$ ), number of authors ( 1 or $>1$ ), years of publication (before or after January 1, 2008), reports of trial dates (yes or no), baseline comparison of participants (yes or no), reports of adverse events (yes or no), follow-up periods ( $\leq 14$ days or $>14$ days), GXN daily dosages $(<20 \mathrm{~mL}, 20 \mathrm{~mL}$, $>20 \mathrm{~mL}$ ), different angina types, and different treatments including GXN monotherapy versus control treatment, GXN + control versus control, and GXN mixed treatment + control versus control. There was no statistically significant difference between ORs of these subgroups.

3.6. Sensitivity Analysis. When the improvement criteria were raised to the significant level from the basic level, the overall results remained effective (i.e., OR $>1$ ) and statistically significant. The OR of overall SYMPTOMS decreased from 3.32 to 1.75 (95\% CI: $[1.54,1.98], Z=8.65, P<0.0001$ ). The OR of overall ECG decreased from 2.59 to 1.84 (95\% CI: $[1.59,2.14], Z=8.06, P<0.0001)$. There was a statistically significant correlation between the changes in ORs of SYMPTOMS and ECG outcomes (tau $=0.2971$, $P=0.0089$ ). When study [33] with maximum GXN dosage was excluded, there was no statistically significant difference between ORs of groups in both SYMPTOMS and ECG data.

3.7. Metaregression. Table 5 shows the results of metaregression between $\log \mathrm{OR}$ and study characteristics. There seemed to be no statistically significant relationship between GXN's efficacy and study characteristics, except that followup periods made a significant difference $(P=0.0093)$ on the $\log$ OR with ECG data.

3.8. Risk of Bias Across Studies. Visual assessment of funnel plots (Figure 4) found obvious asymmetry, indicating that there were publication biases in the results of both SYMPTOMS and ECG. Egger's test (SYMPTOMS: $t=2.0555$, $P=0.0440$; ECG: $t=0.9358, P=0.3556)$ and Begg's test (SYMPTOMS: $z=0.1898, P=0.0257$; ECG: $z=0.2571, P=$ $0.0236)$ detected statistically significant publication biases.
Trim-and-fill method found that there were 24 missing studies for SYMPTOMS and 13 missing studies for ECG on the left side of the corresponding funnel plots.

3.9. Adverse Events. As shown in Table 6, the most frequently reported adverse event of GXN was headache. All adverse effects were minor or well tolerated as they did not cause dropouts except in one study [31] where six participants dropped out because of the adverse effects. Headache, epigastria discomfort, and palpitation were noted as the top three adverse effects of drugs in control group. Adverse effects of GXN were less than those of control drugs in the number of types, severity, and frequency.

\section{Discussion}

This study provides the first comprehensive, up-to-date, and PRISMA-compliant systematic review on the efficacy of GXN in treating angina pectoris. Among 65 included RCTs with 6064 participants, overall ORs of SYMPTOMS and ECG were 3.32 (95\% CI: $[2.72,4.04])(P<0.0001)$ and 2.59 (95\% CI: $[2.14,3.15])(P<0.0001)$, respectively. Subgroup analysis also found statistical significance in the differences between GXN treatment group and control group in testing GXN monotherapy and adjunctive therapy. These results indicated that GXN treatment is effective in treating angina pectoris.

The results of this meta-analysis were robust as shown in subgroup analysis, sensitivity analysis, and metaregression on various parameters including sample sizes, follow-up periods, daily dosages of GXN, types of angina pectoris, and the quality scores of RCTs. Although funnel plots, Begg's test, Egger's test, and trim-and-fill method found publication biases, the overall effects would still favor GXN treatment after enough number of less favorable studies were published to restore the symmetry of funnel plots.

The efficacy of GXN in both monotherapy and adjunctive therapy of angina pectoris exemplifies potential uses of chemical components of GXN as one of the herbal products 
TABLE 2: Quality assessment of included studies.

\begin{tabular}{|c|c|c|c|c|c|c|c|c|c|c|c|c|c|c|c|}
\hline Study & $\mathrm{Cl}$ & $\mathrm{C} 2$ & $\mathrm{C} 3$ & $\mathrm{C} 4$ & $\mathrm{C} 5$ & C6 & $\mathrm{C} 7$ & $\mathrm{C} 8$ & Comparable & Random & Blind & Dropout & $\mathrm{AE}$ & Jadad & $M$ \\
\hline Chen 2009 & Low & High & High & High & Low & Low & High & High & 1 & 1 & 0 & 1 & 0 & 2 & 3 \\
\hline Chen et al. 2011 & Low & Unclear & High & High & Low & Low & High & High & 0 & 1 & 0 & 1 & 0 & 2 & 2 \\
\hline Chen 2006 & Low & High & High & High & Low & Low & Low & Low & 1 & 1 & 0 & 1 & 1 & 2 & 4 \\
\hline Cheng and Zeng 2010 & Low & High & High & High & Low & Low & High & High & 1 & 1 & 0 & 1 & 0 & 2 & 3 \\
\hline Cheng et al. 2011 & Low & High & High & High & Low & Low & Low & Low & 1 & 1 & 0 & 1 & 1 & 2 & 4 \\
\hline Dong 2009 & Low & High & High & High & Low & Low & Unclear & High & 0 & 1 & 0 & 1 & 0.5 & 2 & 2.5 \\
\hline Fu and Meng 2011 & Low & High & High & High & Low & Low & High & High & 1 & 1 & 0 & 1 & 0 & 2 & 3 \\
\hline Fu et al. 2010 & Low & High & High & High & Low & Low & High & High & 1 & 1 & 0 & 1 & 0 & 2 & 3 \\
\hline Gao et al. 2005 & Low & Low & High & High & Low & Low & Low & Low & 1 & 1 & 1 & 1 & 1 & 3 & 5 \\
\hline Gong et al. 2009 & Low & High & High & High & Low & Low & Low & Low & 1 & 1 & 0 & 1 & 1 & 2 & 4 \\
\hline He 2007 & Low & High & High & High & Low & Low & High & High & 1 & 1 & 0 & 1 & 0 & 2 & 3 \\
\hline He 2009 & Low & High & High & High & Low & Low & Low & Low & 1 & 1 & 0 & 1 & 1 & 2 & 4 \\
\hline Hou and Gao 2009 & Low & High & High & High & Low & Low & Low & Low & 1 & 1 & 0 & 1 & 1 & 2 & 4 \\
\hline Huang et al. 2011 & Low & High & High & High & Low & Low & Low & Low & 1 & 1 & 0 & 1 & 1 & 2 & 4 \\
\hline Jiang et al. 2010 & Low & High & High & High & Low & Low & High & High & 1 & 1 & 0 & 1 & 0 & 2 & 3 \\
\hline Jiang 2009 & Low & High & High & High & Low & Low & Low & Low & 1 & 1 & 0 & 1 & 1 & 2 & 4 \\
\hline Jiang et al. 2010 & Low & High & High & High & Low & Low & Low & Low & 0 & 1 & 0 & 1 & 1 & 2 & 3 \\
\hline Kong 2009 & Low & High & High & High & Low & Low & Unclear & Low & 1 & 1 & 0 & 1 & 1 & 2 & 4 \\
\hline Lan et al. 2006 & Low & High & High & High & Low & Low & Low & Low & 1 & 1 & 0 & 1 & 1 & 2 & 4 \\
\hline Li and Jia 2011 & Low & High & High & High & Low & Low & High & High & 1 & 1 & 0 & 1 & 0 & 2 & 3 \\
\hline Li and Lei 2005 & Low & High & High & High & Low & Low & Low & Low & 0 & 1 & 0 & 1 & 1 & 2 & 3 \\
\hline Li et al. 2009 & Low & Low & Low & Low & Low & Low & High & High & 1 & 1 & 2 & 1 & 0 & 4 & 5 \\
\hline Li and Ran 2009 & Low & High & High & High & Low & Low & Low & Low & 1 & 1 & 0 & 1 & 1 & 2 & 4 \\
\hline Li 2004 & Low & High & High & High & Low & Low & High & Low & 1 & 1 & 0 & 1 & 0 & 2 & 3 \\
\hline Liang and Feng 2010 & Low & High & High & High & Low & Low & High & High & 1 & 1 & 0 & 1 & 0 & 2 & 3 \\
\hline Liu 2004 & Low & High & High & High & Low & Low & Low & Low & 1 & 1 & 0 & 1 & 1 & 2 & 4 \\
\hline Liu and Li 2007 & Low & High & High & High & Low & Low & High & High & 0 & 1 & 0 & 1 & 1 & 2 & 3 \\
\hline Liu 2005 & Low & High & High & High & Low & Low & High & High & 1 & 1 & 0 & 1 & 0 & 2 & 3 \\
\hline Liu 2011 & Low & High & High & High & Low & Low & High & High & 1 & 1 & 0 & 1 & 0 & 2 & 3 \\
\hline Lu et al. 2006 & Low & High & High & High & Low & Low & High & High & 1 & 1 & 0 & 1 & 0 & 2 & 3 \\
\hline Ma and Peng 2008 & Low & High & High & High & Low & Low & Low & Low & 1 & 1 & 0 & 1 & 0 & 2 & 3 \\
\hline Nie and Chen 2007 & Low & High & High & High & Low & Low & High & High & 1 & 1 & 0 & 1 & 0 & 2 & 3 \\
\hline Qiao and Wu 2004 & Low & High & High & High & Low & Low & Low & Low & 1 & 1 & 0 & 1 & 1 & 2 & 4 \\
\hline Song 2010 & Low & High & High & High & Low & Low & Low & Unclear & 0 & 1 & 0 & 1 & 0.5 & 2 & 2.5 \\
\hline Su 2009 & Low & Unclear & High & High & Low & Low & Low & Low & 0 & 1 & 0 & 1 & 1 & 2 & 3 \\
\hline Sun 2010 & Low & High & High & High & Low & Low & High & High & 1 & 1 & 0 & 1 & 0 & 2 & 3 \\
\hline Sun et al. 2006 & Low & High & High & High & Low & Low & High & Unclear & 0 & 1 & 0 & 1 & 0 & 2 & 2 \\
\hline Tian and Wu 2006 & Low & High & High & High & Low & Low & Low & Low & 1 & 1 & 0 & 1 & 1 & 2 & 4 \\
\hline Wan and Xu 2009 & Low & High & High & High & Low & Low & Low & Low & 1 & 1 & 0 & 1 & 1 & 2 & 4 \\
\hline Wang 2007 & Low & High & High & High & Low & Low & Low & High & 1 & 1 & 0 & 1 & 1 & 2 & 4 \\
\hline Wang 2011 & Low & High & High & High & Low & Low & Low & Low & 1 & 1 & 0 & 1 & 1 & 2 & 4 \\
\hline Wang 2011 & Low & High & High & High & Low & Low & Low & Low & 1 & 1 & 0 & 1 & 1 & 2 & 4 \\
\hline Wang and Ji 2008 & Low & High & High & High & Low & Low & High & High & 0 & 1 & 0 & 1 & 0 & 2 & 2 \\
\hline Wang and Sun 2007 & Low & High & High & High & Low & Low & High & Unclear & 0 & 1 & 0 & 1 & 0 & 2 & 2 \\
\hline Wang 2005 & Low & High & High & High & Low & Low & High & High & 1 & 1 & 0 & 1 & 0 & 2 & 3 \\
\hline Wang 2010 & Low & High & High & High & Low & Low & Low & Low & 1 & 1 & 0 & 1 & 1 & 2 & 4 \\
\hline Wang 2005 & Low & High & High & High & Low & Low & High & Unclear & 0 & 1 & 0 & 1 & 0 & 2 & 2 \\
\hline Wang 2005 & Low & Low & Unclear & High & Low & Low & Low & Low & 1 & 1 & 0 & 1 & 1 & 2 & 4 \\
\hline Wang et al. 2011 & Low & High & High & High & Low & Low & Low & Low & 1 & 1 & 0 & 1 & 1 & 2 & 4 \\
\hline Wu et al. 2008 & Low & High & High & High & Low & Low & High & Unclear & 1 & 1 & 0 & 1 & 0 & 2 & 3 \\
\hline
\end{tabular}


TABLE 2: Continued.

\begin{tabular}{|c|c|c|c|c|c|c|c|c|c|c|c|c|c|c|c|}
\hline Study & $\mathrm{C} 1$ & $\mathrm{C} 2$ & $\mathrm{C} 3$ & $\mathrm{C} 4$ & $\mathrm{C} 5$ & C6 & $\mathrm{C} 7$ & $\mathrm{C} 8$ & Comparable & Random & Blind & Dropout & $\mathrm{AE}$ & Jadad & $M$ \\
\hline Wu et al. 2011 & Low & High & High & High & Low & Low & High & High & 1 & 1 & 0 & 1 & 0 & 2 & 3 \\
\hline Xia 2011 & Low & Low & Unclear & High & Low & Low & High & Unclear & 1 & 1 & 0 & 1 & 0 & 2 & 3 \\
\hline Yang and Ma 2008 & Low & Low & Unclear & High & Low & Low & High & High & 1 & 1 & 0 & 1 & 0 & 2 & 3 \\
\hline Ye et al. 2008 & Low & High & High & High & Low & Low & Low & Low & 0 & 1 & 0 & 1 & 1 & 2 & 3 \\
\hline Yu and Wang 2009 & Low & High & High & High & Low & Low & High & High & 1 & 1 & 0 & 1 & 0 & 2 & 3 \\
\hline Yuan 2005 & Low & High & High & High & Low & Low & High & High & 1 & 1 & 0 & 1 & 0 & 2 & 3 \\
\hline Zhang 2005 & Low & High & High & High & Low & Low & High & High & 1 & 1 & 0 & 1 & 0 & 2 & 3 \\
\hline Zhang 2010 & Low & High & High & High & Low & Low & Low & Low & 1 & 1 & 0 & 1 & 1 & 2 & 4 \\
\hline Zhang 2004 & Low & High & High & High & Low & Low & Low & Low & 1 & 1 & 0 & 1 & 1 & 2 & 4 \\
\hline Zhang 2004 & Low & High & High & High & Low & Low & Low & High & 0 & 1 & 0 & 1 & 1 & 2 & 3 \\
\hline Zhao et al. 2010 & Low & High & High & High & Low & Low & Low & Low & 1 & 1 & 0 & 1 & 1 & 2 & 4 \\
\hline Zhao and An 2008 & Low & High & High & High & Low & Low & Low & Low & 1 & 1 & 0 & 1 & 1 & 2 & 4 \\
\hline Zhao 2010 & Low & High & High & High & Low & Low & High & Unclear & 1 & 1 & 0 & 1 & 0 & 2 & 3 \\
\hline Zhong et al. 2007 & Low & High & High & High & Low & Low & High & High & 0 & 1 & 0 & 1 & 0 & 2 & 2 \\
\hline Zhu 2005 & Low & High & High & High & Low & Low & Unclear & Unclear & 0 & 1 & 0 & 1 & 0.5 & 2 & 2.5 \\
\hline
\end{tabular}

$\mathrm{C} 1$ is random sequence generation for selection bias; $\mathrm{C} 2$ is allocation concealment for selection bias; $\mathrm{C} 3$ is blinding of participants and personnel for performance bias; $\mathrm{C} 4$ is blinding of outcome assessment (patient-reported outcomes) for detection bias; C5 is blinding of outcome assessment (SYMPTOMS) for detection bias; $\mathrm{C} 6$ is incomplete outcome data addressed for attrition bias; $\mathrm{C} 7$ is reporting bias for selecting reporting; $\mathrm{C} 8$ is other sources of bias for other bias; Comparable is participants in treat group and control group comparable; Random is study described as randomized; Blind is study described as blinding; Dropout is withdrawals and dropouts of participants; AE is the adverse effects; Low is low risk of bias; High is high risk of bias; Unclear is unclear risk of bias.

TABLE 3: Subgroups and sensitivity analysis on SYMPTOMS outcomes.

\begin{tabular}{|c|c|c|c|c|c|c|c|c|c|c|c|}
\hline & Group & $\begin{array}{l}\text { Number of } \\
\text { RCTs }\end{array}$ & $\begin{array}{l}\text { Number of } \\
\text { participants }\end{array}$ & OR & Wilcoxon test & $95 \%$ CI & $\mathrm{Z}$ & $P($ eff $)$ & $I^{2}$ & $\chi^{2}$ & $P$ (het) \\
\hline \multirow{2}{*}{$M$ score } & $\leq 3$ & 40 & 3625 & 3.21 & $W=546$ & $2.36,4.35$ & 7.46 & $<0.0001$ & $54 \%$ & 0.50 & $<0.0001$ \\
\hline & $>3$ & 25 & 2439 & 3.51 & $P=0.5395$ & $2.78,4.43$ & 10.50 & $<0.0001$ & $0 \%$ & 0 & 0.9858 \\
\hline \multirow{2}{*}{ Sample size } & $<93$ & 39 & 2772 & 3.22 & $W=445.5$ & $2.59,4.01$ & 10.51 & $<0.0001$ & $0 \%$ & 0 & 0.6150 \\
\hline & $\geq 93$ & 26 & 3292 & 3.37 & $P=0.4140$ & $2.39,4.76$ & 6.89 & $<0.0001$ & $60 \%$ & 0.47 & $<0.0001$ \\
\hline \multirow{2}{*}{ Number of authors } & 1 & 27 & 2485 & 3.18 & $W=1189$ & $2.39,4.24$ & 7.92 & $<0.0001$ & $28 \%$ & 0.16 & 0.1253 \\
\hline & $>1$ & 38 & 3579 & 3.40 & $P=0.7558$ & $2.60,4.46$ & 8.87 & $<0.0001$ & $44 \%$ & 0.30 & 0.0031 \\
\hline \multirow{2}{*}{ Publication year } & $\leq 2008$ & 31 & 2495 & 3.80 & $W=441.5$ & $3.01,4.81$ & 11.19 & $<0.0001$ & $1 \%$ & 0.01 & 0.2929 \\
\hline & $>2008$ & 34 & 3569 & 2.94 & $P=0.2642$ & $2.20,3.93$ & 7.32 & $<0.0001$ & $48 \%$ & 0.34 & 0.0016 \\
\hline \multirow{2}{*}{ Trial date report } & Reported & 51 & 4793 & 3.19 & $W=2112.5$ & $2.57,3.95$ & 10.52 & $<0.0001$ & $36 \%$ & 0.21 & 0.0189 \\
\hline & Not reported & 14 & 1271 & 3.84 & $P=1$ & $2.33,6.33$ & 5.28 & $<0.0001$ & $47 \%$ & 0.40 & 0.0254 \\
\hline \multirow{2}{*}{$\begin{array}{l}\text { Baseline } \\
\text { comparison }\end{array}$} & Reported & 50 & 4808 & 3.56 & $W=2112.5$ & $2.84,4.45$ & 11.10 & $<0.0001$ & $40 \%$ & 0.25 & 0.0057 \\
\hline & Not reported & 15 & 1256 & 2.53 & $P=1$ & $1.75,3.68$ & 4.89 & $<0.0001$ & $14 \%$ & 0.08 & 0.1545 \\
\hline \multirow{2}{*}{$\begin{array}{l}\text { Adverse } \\
\text { events }\end{array}$} & Reported & 31 & 2947 & 3.20 & $W=1006$ & $2.58,3.97$ & 10.59 & $<0.0001$ & $0 \%$ & 0 & 0.4304 \\
\hline & Not reported & 34 & 3117 & 3.48 & $P=0.4678$ & $2.53,4.78$ & 7.68 & $<0.0001$ & $51 \%$ & 0.44 & 0.0003 \\
\hline \multirow{2}{*}{$\begin{array}{l}\text { Follow-up } \\
\text { period (day) }\end{array}$} & $\leq 14$ & 48 & 4461 & 3.38 & $W=440$ & $2.75,4.16$ & 11.51 & $<0.0001$ & $28 \%$ & 0.14 & 0.1321 \\
\hline & $>14$ & 17 & 1603 & 3.05 & $P=0.6382$ & $1.81,5.16$ & 4.18 & $<0.0001$ & $61 \%$ & 0.71 & 0.0005 \\
\hline GXN daily & $6-200 \mathrm{~mL}$ & 65 & 6064 & 3.32 & $W=2059$ & $2.72,4.04$ & 11.93 & $<0.0001$ & $37 \%$ & 0.23 & 0.0030 \\
\hline Dosage $(\mathrm{mL})$ & 6-30 mL & 64 & 6008 & 3.34 & $P=0.9231$ & $2.73,4.07$ & 11.83 & $<0.0001$ & $38 \%$ & 0.24 & 0.0025 \\
\hline GXN daily & $<20$ & 4 & 352 & 3.42 & $\chi^{2}=0.4290$ & $1.48,7.91$ & 2.88 & 0.0040 & $38 \%$ & 0.28 & 0.1717 \\
\hline \multirow{2}{*}{ Dosage $(\mathrm{mL})$} & 20 & 45 & 4235 & 3.16 & $\mathrm{df}=2$ & $2.45,4.07$ & 8.85 & $<0.0001$ & $46 \%$ & 0.33 & 0.0004 \\
\hline & $>20$ & 16 & 1477 & 3.87 & $P=0.8069$ & $2.84,5.29$ & 8.51 & $<0.0001$ & $0 \%$ & 0 & 0.8315 \\
\hline \multirow{3}{*}{ Types of angina } & Stable & 4 & 374 & 3.42 & $\chi^{2}=0.9900$ & $1.89,6.21$ & 4.05 & $<0.0001$ & $0 \%$ & 0 & 0.7151 \\
\hline & Unstable & 31 & 2892 & 3.07 & $\mathrm{df}=2$ & $2.26,4.16$ & 7.18 & $<0.0001$ & $47 \%$ & 0.34 & 0.0013 \\
\hline & Angina & 30 & 2798 & 3.61 & $P=0.6096$ & $2.72,4.81$ & 8.81 & $<0.0001$ & $32 \%$ & 0.19 & 0.1179 \\
\hline \multirow{2}{*}{ Improvement } & $>50 \%$ & 65 & 6064 & 3.32 & $W=02.5$ & $2.72,4.04$ & 11.93 & $<0.0001$ & $37 \%$ & 0.23 & 0.0030 \\
\hline & $>80 \%$ & 63 & 5856 & 1.75 & $P<0.0001$ & $1.54,1.98$ & 8.65 & $<0.0001$ & $25 \%$ & 0.06 & 0.0557 \\
\hline GXN & 1 & 6 & 408 & 3.19 & $\chi^{2}=0.4891$ & $1.86,5.49$ & 4.21 & $<0.0001$ & $0 \%$ & 0 & 0.8454 \\
\hline $\mathrm{GXN}+\mathrm{CG}$ & 2 & 49 & 4681 & 3.43 & $\mathrm{df}=2$ & $2.81,4.19$ & 12.07 & $<0.0001$ & $21 \%$ & 0.11 & 0.1177 \\
\hline $\begin{array}{l}\text { GXN + CG + } \\
\text { additional }\end{array}$ & 3 & 10 & 975 & 3.07 & $P=0.7830$ & $1.47,6.41$ & 2.99 & 0.00228 & $72 \%$ & 0.98 & $<0.0001$ \\
\hline
\end{tabular}

CI is confidence interval; $Z$ and $P$ (eff) are statistical terms for evaluating overall effect; $I^{2}, \chi^{2}$, and $P$ (het) are statistical terms for assessing heterogeneity among studies. 


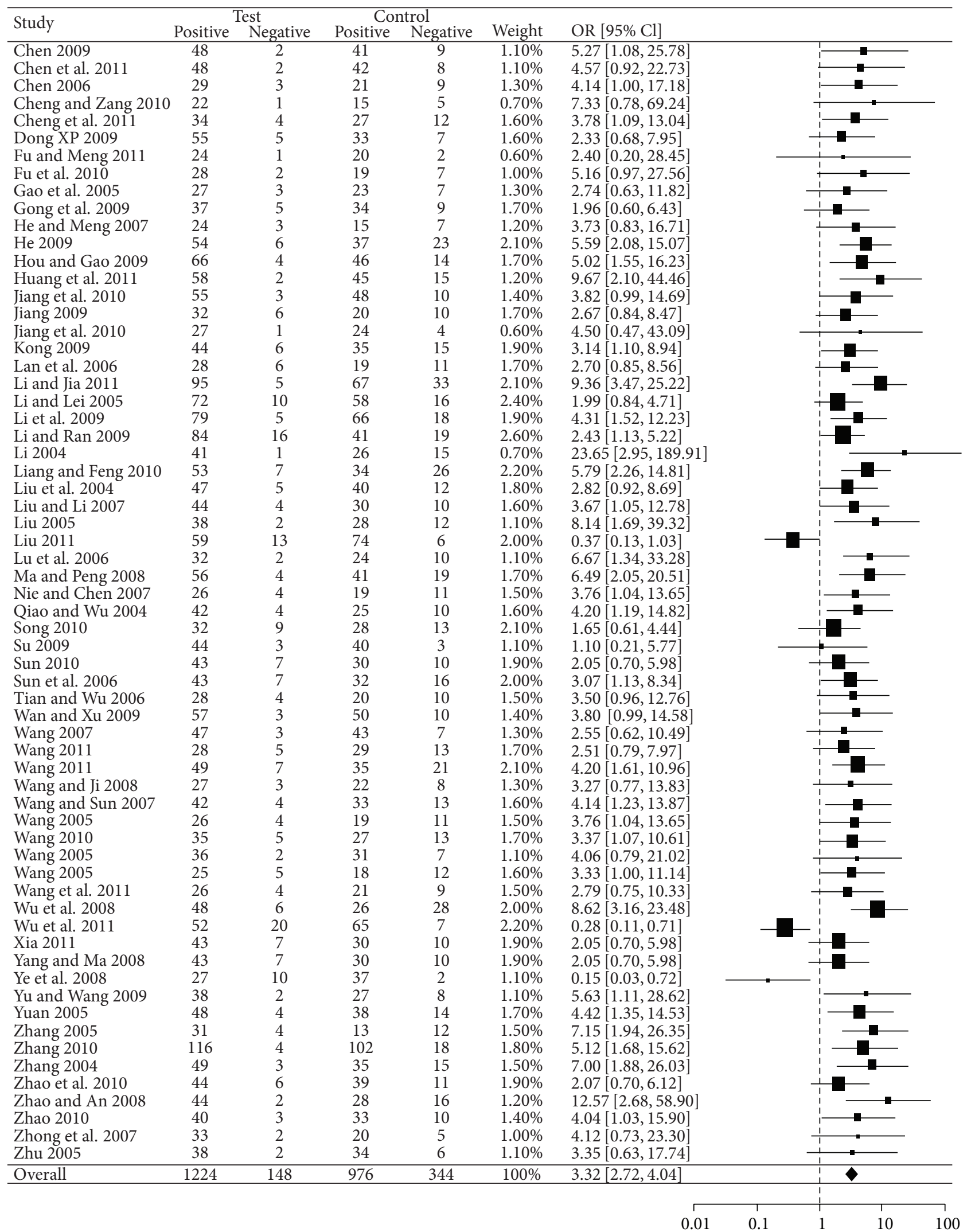

FIGURE 2: Forest plot of outcome measure SYMPTOMS. 


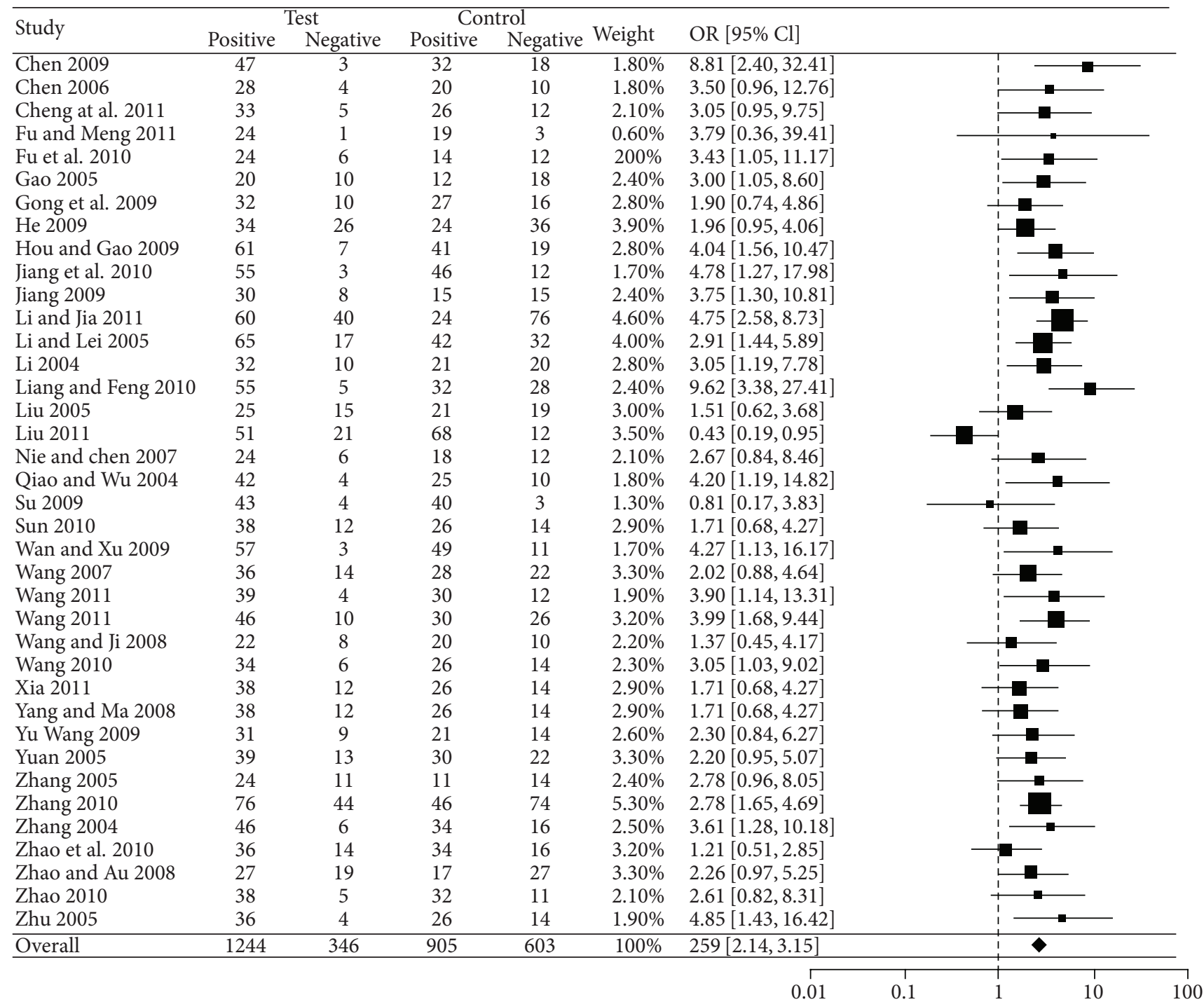

FIGURE 3: Forest plot of outcome measure ECG.

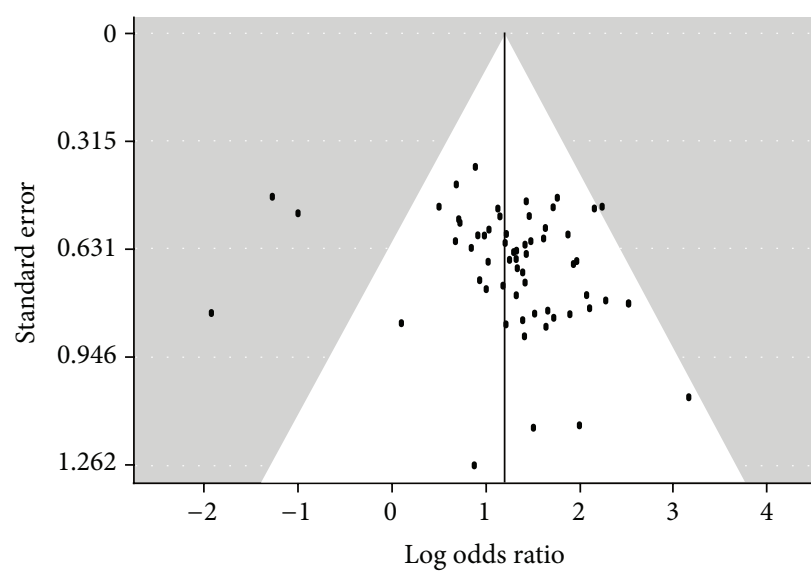

(a)

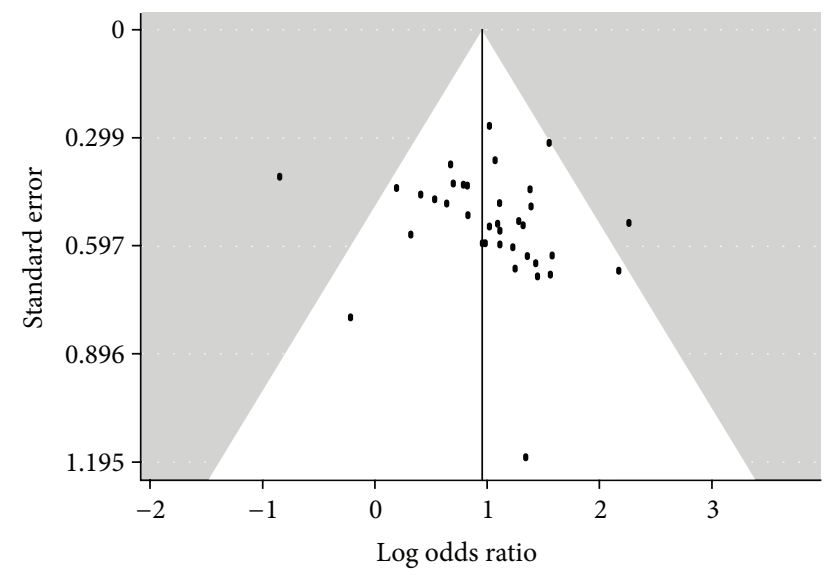

(b)

FIGURE 4: Funnel plots of (a) the included studies with SYMPTOMS data and (b) the included studies with ECG data. 
TABLE 4: Subgroups and sensitivity analysis on ECG outcomes.

\begin{tabular}{|c|c|c|c|c|c|c|c|c|c|c|c|}
\hline & Group & $\begin{array}{c}\text { Number of } \\
\text { RCTs }\end{array}$ & $\begin{array}{l}\text { Number of } \\
\text { participants }\end{array}$ & OR & Wilcoxon test & $95 \%$ CI & $Z$ & $P($ eff $)$ & $I^{2}$ & $\chi^{2}$ & $P$ (het) \\
\hline \multirow{2}{*}{$M$ score } & $\leq 3$ & 21 & 1995 & 2.47 & $W=149$ & $1.79,3.41$ & 5.53 & $<0.0001$ & $52 \%$ & 0.28 & 0.0025 \\
\hline & $>3$ & 17 & 1709 & 2.71 & $P=0.3945$ & $2.17,3.39$ & 8.77 & $<0.0001$ & $0 \%$ & 0 & 0.9136 \\
\hline \multirow{2}{*}{ Sample size } & $<93$ & 23 & 1734 & 2.42 & $W=127$ & $1.93,3.02$ & 7.72 & $<0.0001$ & $0 \%$ & 0 & 0.9776 \\
\hline & $\geq 93$ & 15 & 1970 & 2.86 & $P=0.1789$ & $1.94,4.21$ & 5.33 & $<0.0001$ & $67 \%$ & 0.37 & 0.0002 \\
\hline \multirow{2}{*}{ Number of authors } & 1 & 19 & 1872 & 2.30 & $W=140$ & $1.72,3.08$ & 5.65 & $<0.0001$ & $41 \%$ & 0.16 & 0.0358 \\
\hline & $>1$ & 19 & 1832 & 2.98 & $P=0.2428$ & $2.34,3.80$ & 8.81 & $<0.0001$ & $13 \%$ & 0.04 & 0.4435 \\
\hline \multirow{2}{*}{ Publication year } & $\leq 2008$ & 15 & 1268 & 2.49 & $W=200$ & $1.94,3.20$ & 7.17 & $<0.0001$ & $0 \%$ & 0 & 0.9538 \\
\hline & $>2008$ & 23 & 2436 & 2.68 & $P=0.4200$ & $1.99,3.61$ & 6.47 & $<0.0001$ & $52 \%$ & 0.26 & 0.0025 \\
\hline \multirow{2}{*}{ Trial date report } & Reported & 31 & 3069 & 2.43 & $W=57$ & $1.97,3.00$ & 8.29 & $<0.0001$ & $34 \%$ & 0.12 & 0.0511 \\
\hline & Not reported & 7 & 635 & 3.67 & $P=0.0548$ & $2.36,5.70$ & 5.79 & $<0.0001$ & $8 \%$ & 0.03 & 0.5363 \\
\hline \multirow{2}{*}{$\begin{array}{l}\text { Baseline } \\
\text { comparison }\end{array}$} & Reported & 34 & 3318 & 2.64 & $W=85$ & $2.14,3.24$ & 9.20 & $<0.0001$ & $35 \%$ & 0.12 & 0.0513 \\
\hline & Not reported & 4 & 386 & 2.29 & $P=0.4325$ & $1.25,4.19$ & 2.68 & 0.0074 & $23 \%$ & 0.09 & 0.2200 \\
\hline \multirow{2}{*}{$\begin{array}{l}\text { Adverse } \\
\text { events }\end{array}$} & Reported & 19 & 1955 & 2.67 & $W=192$ & $2.16,3.30$ & 9.14 & $<0.0001$ & $0 \%$ & 0 & 0.8792 \\
\hline & Not reported & 19 & 1749 & 2.54 & $P=0.7480$ & $1.80,3.59$ & 5.28 & $<0.0001$ & $54 \%$ & 0.31 & 0.0020 \\
\hline Follow-up & $\leq 14$ & 27 & 2528 & 2.83 & $W=129$ & $2.34,3.42$ & 10.67 & $<0.0001$ & $2 \%$ & 0 & 0.7120 \\
\hline period (day) & $>14$ & 11 & 1176 & 2.21 & $P=0.5407$ & $1.37,3.57$ & 3.27 & $<0.0001$ & $65 \%$ & 0.39 & 0.0024 \\
\hline GXN daily & $6-200 \mathrm{~mL}$ & 38 & 3704 & 2.59 & $W=707.5$ & $2.14,3.15$ & 9.68 & $<0.0001$ & $32 \%$ & 0.11 & 0.0539 \\
\hline dosage (mL) & $6-30 \mathrm{~mL}$ & 37 & 3648 & 2.58 & $P=0.9662$ & $2.12,3.14$ & 9.45 & $<0.0001$ & $33 \%$ & 0.1175 & 0.0448 \\
\hline GXN daily & $<20$ & 4 & 352 & 1.89 & $\chi^{2}=3.4288$, & $1.00,3.55$ & 1.96 & 0.0497 & $27 \%$ & 0.1148 & 0.2425 \\
\hline \multirow{2}{*}{ dosage $(\mathrm{mL})$} & 20 & 24 & 2324 & 2.80 & $\mathrm{df}=2$ & $2.14,3.66$ & 7.51 & $<0.0001$ & $43 \%$ & 0.1820 & 0.0246 \\
\hline & $>20$ & 10 & 1028 & 2.53 & $P=0.1801$ & $1.85,3.46$ & 5.81 & $<0.0001$ & $14 \%$ & 0.0365 & 0.5413 \\
\hline \multirow{3}{*}{ Types of angina } & Stable & 4 & 374 & 3.03 & $\chi^{2}=0.7010$ & $1.80,5.09$ & 4.18 & $<0.0001$ & $0 \%$ & 0 & 0.6688 \\
\hline & Unstable & 16 & 1676 & 2.48 & $\mathrm{df}=2$ & $1.95,3.15$ & 7.42 & $<0.0001$ & $10 \%$ & 0.02 & 0.2332 \\
\hline & Angina & 18 & 1654 & 2.60 & $P=0.7043$ & $1.87,3.61$ & 5.68 & $<0.0001$ & $46 \%$ & 0.22 & 0.0191 \\
\hline \multirow{2}{*}{ Improvement } & $>50 \%$ & 38 & 3704 & 2.59 & $W=1050$ & $2.14,3.15$ & 9.68 & $<0.0001$ & $32 \%$ & 0.11 & 0.0539 \\
\hline & $>80 \%$ & 38 & 3704 & 1.84 & $P<0.0001$ & $1.59,2.14$ & 8.06 & $<0.0001$ & $0 \%$ & 0 & 0.8367 \\
\hline GXN & 1 & 3 & 188 & 3.15 & $\chi^{2}=1.6604$ & $1.71,5.81$ & 3.68 & 0.0002 & $0 \%$ & 0 & 0.9202 \\
\hline $\mathrm{GXN}+\mathrm{CG}$ & 2 & 29 & 2963 & 2.68 & $\mathrm{df}=2$ & $2.10,3.41$ & 7.98 & $<0.0001$ & $42 \%$ & 0.17 & 0.0157 \\
\hline $\begin{array}{l}\mathrm{GXN}+\mathrm{CG}+ \\
\text { additional }\end{array}$ & 3 & 6 & 553 & 2.09 & $P=0.4360$ & $1.45,3.01$ & 3.94 & $<0.0001$ & $0 \%$ & 0 & 0.6382 \\
\hline
\end{tabular}

$\mathrm{CI}$ is confidence interval; $Z$ and $P$ (eff) are statistical terms for evaluating overall effect; $I^{2}, \chi^{2}$, and $P$ (het) are statistical terms for assessing heterogeneity among studies.

TABLE 5: Metaregression analysis of the relationship between outcomes and the study characteristics.

\begin{tabular}{|c|c|c|c|c|c|c|}
\hline $\log$ OR & Number of RCTs & Number of participants & Factor & Coefficient & $z$ & $P$ \\
\hline \multirow{8}{*}{ SYMPTOMS } & \multirow{8}{*}{65} & \multirow{8}{*}{6064} & $M$ score & 0.0663 & 0.4378 & 0.6615 \\
\hline & & & Sample size & -0.0013 & -0.4955 & 0.6203 \\
\hline & & & Number of authors & -0.0466 & -0.6283 & 0.5298 \\
\hline & & & Publication year & -0.0838 & -1.9158 & 0.0554 \\
\hline & & & Trial date report & -0.1931 & -0.7634 & 0.4453 \\
\hline & & & Baseline comparison & 0.3299 & 1.3376 & 0.1810 \\
\hline & & & Adverse events & -0.0965 & -0.4646 & 0.6422 \\
\hline & & & Follow-up period & 0.0116 & 0.6126 & 0.5401 \\
\hline \multirow{8}{*}{ ECG } & \multirow{8}{*}{38} & \multirow{8}{*}{3704} & $M$ score & 0.1191 & 0.7160 & 0.4740 \\
\hline & & & Sample size & 0.0006 & 0.2938 & 0.7689 \\
\hline & & & Number of authors & -0.0100 & -0.1071 & 0.9147 \\
\hline & & & Publication year & -0.0180 & -0.4296 & 0.6675 \\
\hline & & & Trial date report & -0.4255 & -1.5606 & 0.1186 \\
\hline & & & Baseline comparison & 0.1520 & 0.4458 & 0.6558 \\
\hline & & & Adverse events & 0.1066 & 0.5300 & 0.5961 \\
\hline & & & Follow-up period & -0.0423 & -2.6000 & 0.0093 \\
\hline
\end{tabular}

that have offered great potentials in developing multitarget agents to treat complex diseases [91]. Experimental studies also showed that the aqueous extracts from both Danshen and Chuanxiong significantly reduced the myocardial infarct size in rat myocardial ischemia/reperfusion injury [92]. As seen from the clinical and experimental findings, GXN seems to be a promising resource for identifying new therapeutic agents or new drug targets [93] in treating angina pectoris. Although subgroup analysis and sensitivity analysis did not suggest any significant factors which would influence 
TABLE 6: Adverse events reported in the included studies.

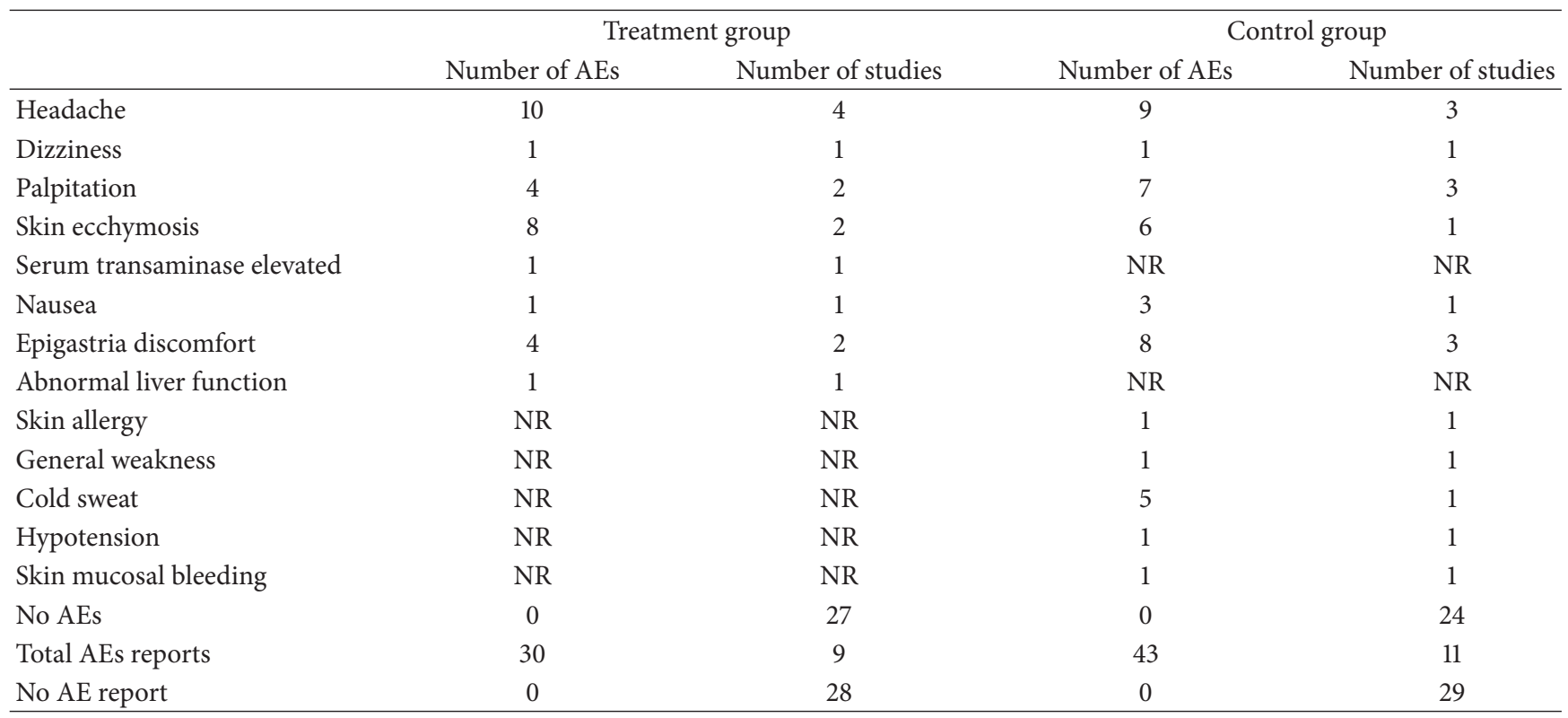

NR: not reported; AEs: adverse events.

the efficacy of GXN, clinical heterogeneity may contribute to heterogeneity of this meta-analysis.

The limitations of this study include small sample sizes and short follow-up periods. The mean sample size was 93, which was lower than 124 as required by an alpha of 0.05 , the proportions of 0.899 for GXN and 0.742 for control group, and a power of 0.8 [94]. The patients of angina pectoris would need long-term treatment [95], but most available RCTs have short follow-up periods.

Another major limitation of this systematic review is the low quality of included studies although most of included RCT reports achieved the average quality of Chinese RCTs $[96,97]$, which is still inadequate. Almost all (63 out of 65 ) studies scored 2 at the Jadad scale, which ranges between 0 and 5. One study [34] reported single blinding and another study [47] reported double blinding. Twenty-four RCTs scored 4 at the $M$ scale and 40 RCTs scored less than 4 at the $M$ scale. There is evidence of the Cochrane Library's tool to show high risks of bias with the aspects of selection bias, performance bias, and detection bias. More than that, less than but almost half of included RCTs (28/65) did not report adverse events, one possible reason of which is high reporting bias for selecting reporting. Safety of GXN intervention cannot be assessed because of incomplete reporting data. Despite the fact that subgroup analysis found no statistically significant differences in ORs of SYMPTOMS and ECG between the RCTs of low and medium $M$ scores, high-quality RCTs would be necessary to further support the efficacy of GXN-based medicines over conventional Western drugs in treating angina pectoris.

Seventy-three out of 6064 participants had AE. The main AEs included headache (19), skin ecchymosis (14), epigastria discomfort (12), and palpitation (11). Headache was the most frequent $\mathrm{AE}$ in this paper. The $\mathrm{AE}$ mechanisms of GXN are not clear and definite. The functions of dilated blood vessels and coronary artery blood circulation activating are possible reasons that lead to adverse events.

According to this meta-analysis, GXN seems to be effective in treating angina pectoris. As GXN contains the herbal extracts from Salvia miltiorrhiza and Ligustrazine, hence DSS, PAC, PAL, CAA, and SAB as the main active ingredients with potential effects on coronary heart disease, angina pectoris, and cardiovascular diseases [98] by enhancing coronary blood flow, improving the myocardial systolic functions, and protecting myocardial cells [99], further clinical, herbal formulation and pharmacological studies are warranted for further research and development.

\section{Conclusion}

This meta-analysis of eligible RCTs provides evidence that GXN is effective in treating angina pectoris. This evidence warrants further RCTs of higher quality, longer follow-up periods, larger sample sizes, and multicentres/multicountries for more extensive subgroup, sensitivity, and metaregression analyses.

\section{Conflict of Interests}

The authors have no conflict of interests.

\section{Authors' Contributions}

S. Leung and M. Lee conceived the meta-analytic assessment of GXN's efficacy. S. Leung, M. Lee, and Y. Jia conducted the study design. G. Cui, X. Huang and M. Lee reviewed the 
pharmacology and potential clinical applications of GXN. Y. Jia and F. Pan searched the databases for RCTs, retrieved the studies, evaluated the quality of the studies, and extracted the data. Y. Jia and S. Leung analyzed the data and wrote the paper. All authors revised, read, and approved the paper.

\section{Acknowledgments}

The work of Y. Jia, F. Pan, and S. Leung was supported by a research Grant from the University of Macau for a study on "Open systematic reviewing of clinical trials" (MYRG190 (Y2-L3)-ICMS11-LSW). The work of G. Cui, X. Huang, M. Lee was supported by a Research Grant from the Science and Technology Development Fund, Macao, for a study on "Mechanistic studies of active compounds from Chinese medicine and their combinations for minimising and treating cardio-cerebrovascular complications" (ref. no. 014/2011/ A1).

\section{References}

[1] T. A. Gaziano, "Cardiovascular disease in the developing world and its cost-effective management," Circulation, vol. 112, no. 23, pp. 3547-3553, 2005.

[2] H. C. Kung, D. L. Hoyert, J. Q. Xu, and S. L. Murphy, "Deaths: final data for 2005. National Vital Statistics Reports," National Center for Health Statistics, vol. 56, no. 10, pp. 1-120, 2008.

[3] Scottish Intercollegiate Guidelines Networks, Management of Stable Angina. A National Clinical Guideline, no. 96, 2007.

[4] S. L. Tan and C. L. Liu, "Clinical observation of compound danshen dripping pill treating stable angina pectoris," Heilongjiang Medical Journal, vol. 14, no. 2, p. 125, 2001.

[5] X. F. Chen, Z. Y. Lou, H. Zhang et al., "Identification of multiple components in Guanxinning injection using hydrophilic interaction liquid chromatography/time-of-flight mass spectrometry and reversed-phase liquid chromatography/time-offlight mass spectrometry," Rapid Communications in Mass Spectrometry, vol. 25, no. 11, pp. 1661-1674, 2011.

[6] T. O. Cheng, "Cardiovascular effects of danshen," International Journal of Cardiology, vol. 14, pp. 9-22, 2007.

[7] Y. Y. Sun, S. F. Li, and C. Quan, "Solubility of ferulic acid and tetramethylpyrazine in supercritical carbon dioxide," Journal of Chemical and Engineering Data, vol. 50, no. 4, pp. 1125-1128, 2005.

[8] H. P. Chen, "Efficacy analysis of Guanxinning injection combined with isosorbide mononitrate treating ischemic heart disease," Chinese Journal of Ethnomedicine and Ethnopharmacy, vol. 10, p. 134, 2009.

[9] Y. L. Shen and Z. L. Sang, "Efficacy analysis of Guanxinning injection treating ischemic heart disease," World Health Digest Medical Periodieal, vol. 8, no. 47, pp. 18-20, 2011.

[10] X. Y. Fu and X. H. Zhao, "Clinical analysis of Guanxinning injection treating ischemic heart disease or cerebral infarction," China Modern Medicine, vol. 16, no. 10, p. 194, 2009.

[11] D. Moher, A. Liberati, J. Tetzlaff, and D. G. Altman, "The PRISMA Group. Preferred reporting items for systematic reviews and meta-analyses: the PRISMA Statement," Plos Medicine, vol. 89, no. 9, pp. 873-880, 2009.
[12] J. M. Wang, "Meta-analysis of Guanxinning injection as adjunctive therapy for unstable Angina pectoris," China Pharmacy, vol. 22, no. 19, pp. 1810-1812, 2011.

[13] A. R. Jadad, R. A. Moore, D. Carroll et al., "Assessing the quality of reports of randomized clinical trials: is blinding necessary?" Controlled Clinical Trials, vol. 17, no. 1, pp. 1-12, 1996.

[14] Y. Jia, F. Huang, S. Zhang, and S. W. Leung, "Is danshen (Salvia miltiorrhiza) dripping pill more effective than isosorbide dinitrate in treating angina pectoris? A systematic review of randomized controlled trials," International Journal of Cardiology, vol. 157, no. 3, pp. 330-340, 2012.

[15] J. Higgins and S. Green, "Analyzing data and undertaking metaanalyses," in Cochrane Handbook For Systematic Reviews of Interventions Version 5.1.0, chapter 8, 2008.

[16] "Nomenclature and criteria for diagnosis of ischemic heart disease. Report of the Joint International Society and Federation of Cardiology/World Health Organization Task Force on standardization of clinical nomenclature," Circulation, vol. 59, no. 3, pp. 607-609, 1979.

[17] T. Lewis, "PROC LOGISTIC: the logistics behind interpreting categorical variable effects," Statistical Data Analysis, pp. 1-7, 2007.

[18] L. Breierova and M. Choudhari, "An introduction to sensitivity analysis," Massachusetts Institute of Technology, vol. 10, pp. 41$107,2001$.

[19] J. P. T. Higgins and S. G. Thompson, "Quantifying heterogeneity in a meta-analysis," Statistics in Medicine, vol. 21, no. 11, pp. 1539$1558,2002$.

[20] J. A. C. Sterne and M. Egger, "Funnel plots for detecting bias in meta-analysis: guidelines on choice of axis," Journal of Clinical Epidemiology, vol. 54, no. 10, pp. 1046-1055, 2001.

[21] C. B. Begg and M. Mazumdar, "Operating characteristics of a rank correlation test for publication bias," Biometrics, vol. 50, no. 4, pp. 1088-1101, 1994.

[22] M. Egger, G. D. Smith, M. Schneider, and C. Minder, "Bias in meta-analysis detected by a simple, graphical test. Increase in studies of publication bias coincided with increasing use of meta-analysis," British Medical Journal, vol. 316, pp. 629-634, 1997.

[23] S. J. Duval and R. L. Tweedie, "Trim and fill: a simple funnelplot-based method of testing and adjusting for publication bias in meta-analysis," Biometrics, vol. 56, no. 2, pp. 455-463, 2000.

[24] S. G. Thompson and J. P. T. Higgins, "How should metaregression analyses be undertaken and interpreted?" Statistics in Medicine, vol. 21, no. 11, pp. 1559-1573, 2002.

[25] R Development Core Team, " $\mathrm{R}$ : a language and environment for statistical computing," Reference index version 2.12.0. R Foundation for Statistical Computing, Vienna, Austria, 2005, http://www.r-project.org/.

[26] H. P. Chen, "Clinical observation of Guanxinning combined with isosorbide mononitrate in the treatment of Coronary Heart Disease," Chinese Journal of Ethnomedicine and Ethnopharmacy, vol. 10, p. 134, 2009.

[27] R. J. Chen, X. Yang, and X. S. Yang, "Clinical observation of Guanxinning combined with potassium magnesium aspartate in the treatment of coronary artery disease," Contemporary Medicine, vol. 17, p. 87, 2011.

[28] S. G. Chen, "Clinical observation of Guanxinning injection in the treatment of coronary artery disease," Modern Journal of Integrated Traditional Chinese and Western Medicine, vol. 15, pp. 2219-2220, 2006. 
[29] H. Y. Cheng and W. P. Zhang, "The clinical experience with Guanxinning injection in the treatment of elderly diabetic patients with unstable angina," Journal of Bingtuan Medicine, vol. 25, pp. 21-22, 2010.

[30] Y. S. Cheng, R. B. Tan, and D. M. Zhao, "Guanxinning injection in the treatment of unstable angina pectoris: a report of 72 cases," China Foreign Medical Treatment, vol. 8, p. 110, 2011.

[31] X. P. Dong, "Clinical observation of Guanxinning injection in the treatment of angina pectoris: a report of 60 cases," Chinese Journal of Modern Drug Application, vol. 3, pp. 144-145, 2009.

[32] Y. C. Fu and L. Q. Meng, "Clinical observation of Guanxinning injection in the treatment of angina pectoris," Journal of New Chinese Medicine, vol. 43, pp. 8-9, 2011.

[33] Y. W. Fu, C. X. Jiao, Y. H. Shi, and F. Guo, "Clinical observation of Guanxinning injection combined with Shengmai injection in the treatment of Coronary Heart Disease," Journal of Emergency in Traditional Chinese Medicine, vol. 19, pp. 61-62, 2010.

[34] G. Q. Gao, F. Wang, and H. P. Sun, "Clinical observation of Guanxinning injection in the treatment of angina pectoris," $\mathrm{Nei}$ Mongol Journal of Traditional Chinese Medicine, vol. 24, pp. 6-7, 2005.

[35] C. J. Gong, P. J. Wang, and L. M. Huang, "Clinical observation of Xueshuantong combined with Guanxinning injection in the treatment of angina pectoris," Asia-Pacific Traditional Medicine, vol. 5, pp. 52-54, 2009.

[36] H. Y. He, "Clinical observation of Guanxinning injection in the treatment of unstable angina: a report of 27 cases," China Modern Doctor, vol. 45, pp. 43-44, 2007.

[37] Y. J. He, "Clinical observation of atorvastatin combined with Guanxinning injection in the treatment of unstable angina," Chinese Journal of Clinical Rational Drug Use, vol. 2, pp. 4-5, 2009.

[38] G. P. Hou and X. Y. Gao, "Clinical observation of Guanxinning injection and dipyridamole in the treatment of angina pectoris," Journal of Qiqihar Medical College, vol. 30, pp. 158-159, 2009.

[39] Y. Huang, B. Li, D. H. Li, and J. S. Chen, "Clinical observation on low molecular weight heparins calcium combined with Guanxinning and Xuesaitong in cerebral infarction," Journal of Clinical Rational Drug Use, vol. 4, pp. 7-8, 2011.

[40] S. Jiang, Z. Y. Xiong, and F. Z. Yong, "Clinical observation of Guanxinning injection in the treatment of unstable angina pectoris," China Modern Doctor, vol. 48, pp. 125-138, 2010.

[41] S. H. Jiang, "Clinical observation of Guanxinning injection in the treatment of unstable angina: a report of 38 cases," China Medical Herald, vol. 6, p. 105, 2009.

[42] S. L. Jiang, S. Y. Zheng, X. S. Guo, S. H. Yi, and X. Y. Zou, "Clinical observation of Guanxinning injection in the treatment of angina pectoris," China Foreign Medical Treatment, vol. 20, p. $123,2010$.

[43] L. X. Kong, "Clinical observation of Guanxinning injection combined with ferulic sodium in the treatment of unstable angina pectoris," Clinical Medicine, vol. 29, pp. 39-40, 2009.

[44] P. M. Lan, W. B. Luo, and G. M. Weng, "Clinical observation of Guanxinning Injection in the treatment of coronary artery disease and effects on blood lipids," Chinese Medicine Modern Distance Education of China, vol. 4, p. 39, 2006.

[45] C. T. Li and X. Z. Jia, "Clinical observation of Guanxinning injection in the treatment of diabetic patients with angina pectoris: a report of 100 cases," Chinese Journal of Clinical Healthcare, vol. 14, pp. 306-307, 2011.
[46] H. Li and J. P. Lei, "Guanxinning Injection in the treatment of angina pectoris," Journal of Medical Forum, vol. 26, p. 52, 2005.

[47] H. Li, H. Cai, L. Z. Wang, H. Y. Yang, and D. M. Ma, “The improvement of heart function of Guanxinning injection in the patients with angina decubitus," China Medical Herald, vol. 6, 2009.

[48] L. Li and G. X. Ran, "Clinical observation Guanxinning Injection adjuvant therapy for angina pectoris: a report of 100 cases," Shandong Medical Journal, vol. 49, pp. 97-98, 2009.

[49] X. B. Li, "Clinical observation of low molecular weight heparin combined with Guanxinning injection in the treatment of unstable angina," Heilongjiang Medical Journal, vol. 28, pp. 687688, 2004.

[50] H. Y. Liang and Y. G. Feng, "Clinical observation of low molecular weight heparin combined with Guanxinning injection in the treatment of unstable angina," Medical Innovation of China, vol. 7, pp. 106-107, 2010.

[51] B. Q. Liu, "Clinical observation of low molecular weight heparin and Guanxinning injection in the treatment of unstable angina," Medicine Industry Information, vol. 2, pp. 77-124, 2004.

[52] L. Liu and M. Z. Li, "Clinical observation Guanxinning Injection in the treatment of angina pectoris: a report of 48 cases," Jiangxi Journal of Traditional Chinese Medicine, vol. 38, pp. 2324, 2007.

[53] Y. L. Liu, "Clinical observation of Guanxinning injection in the treatment of unstable angina pectoris," Chinese Journal of Integrated Traditional and Western Medicine in Intensive and Critical Care, vol. 12, pp. 181-182, 2005.

[54] Z. H. Liu, "Qi decoction in the treatment of angina pectoris: a report of 80 cases," Journal of Traditional Chinese Medicine, vol. 26, pp. 709-710, 2011.

[55] C. X. Lu, J. Zhang, and B. Tang, "Guanxinning injection combined with isosorbide dinitrate treating the aged patients with ischemic heart disease angina pectoris," Journal of Yangtze University (Nature Science Edition), vol. 3, no. 3, p. 230, 2006.

[56] X. Y. Ma and L. W. Peng, "Clinical observation of Guanxinning injection in the treatment of unstable angina," Journal of Modern Clinical Medicine, vol. 34, pp. 41-42, 2008.

[57] Y. B. Nie and H. Y. Chen, "Clinical observation of Guanxinning injection in the treatment of angina pectoris," Journal of Huaihai Medicine, vol. 25, p. 544, 2007.

[58] W. L. Qiao and Z. R. Wu, "Guanxinning injection for stable angina pectoris of coronary heart disease," Journal of Henan University of Chinese Medicine, vol. 19, p. 51, 2004.

[59] G. F. Song, "Analysis study of Diltiazem combined with Guanxinning in the treatment of unstable angina pectoris," Chronic Pathematology Journal, vol. 12, p. 838, 2010.

[60] X. D. Su, "Clinical observation Guanxinning Injection in the treatment of angina pectoris and myocardial ischemia," Proceeding of Clinical Medicine, vol. 18, pp. 1813-1814, 2009.

[61] S. P. Sun, "Clinical efficacy analysis of Guanxinning combined with Western medicine in the treatment of unstable angina," Jinlin Medical Journal, vol. 31, pp. 325-326, 2010.

[62] Z. H. Sun, P. F. Dong, D. W. Sun, and L. Y. Zhao, "Guanxinning injection in the treatment of angina pectoris: a report of 50 cases," Harbin Medical Journal, vol. 26, pp. 30-31, 2006.

[63] Z. Q. Tian and L. Wu, "Clinical observation of Guanxinning combined with Western medicine in the treatment of unstable angina," Chinese Journal of Cardiovascular Rehabilitation Medicine, vol. 15, pp. 184-186, 2006. 
[64] S. Q. Wan and A. Y. Xu, "Clinical observation of Guanxinning injection in the treatment of unstable angina pectoris," Modern Journal of Integrated Traditional Chinese and Western Medicine, vol. 18, pp. 2016-2017, 2009.

[65] E. Wang, "Clinical observation of Guanxinning injection in the treatment of angina pectoris," Journal of Emergency in Traditional Chinese Medicine, vol. 16, pp. 260-261, 2007.

[66] G. L. Wang, "Clinical observation of Guanxinning injection in the treatment of unstable angina pectoris: a report of 43 cases," Chinese Remedies and Clinics, vol. 11, pp. 1458-1459, 2011.

[67] H. T. Wang and C. M. Yan, "Clinical observation of Guanxinning injection in the treatment of unstable angina pectoris," Chinese Journal of Clinical Research, vol. 24, pp. 792-793, 2011.

[68] J. J. Wang and X. P. Ji, "Clinical observation of Guanxinning injection in the treatment of unstable angina pectoris," Journal of Liaoning University of TCM, vol. 10, pp. 81-82, 2008.

[69] L. J. Wang and X. M. Sun, "Clinical observation of Guanxinning injection in the treatment of unstable angina pectoris," Journal of Qiqihar Medical College, vol. 28, pp. 1350-1351, 2007.

[70] Q. Wang, "Clinical observation of Guanxinning injection in the treatment of angina pectoris," Modern Medicine and Health, vol. 21, pp. 1280-1281, 2005.

[71] Q. Wang, "Clinical observation of Guanxinning injection in the treatment of stable angina pectoris: a report of 40 cases," Yunnan Journal of Traditional Chinese Medicine and Materia Medica, vol. 31, p. 47, 2010.

[72] R. Z. Wang, "Clinical observation of Guanxinning combined with Western medicine in the treatment of unstable angina: a report of 38 cases," The Journal of Medical Theory and Practice, vol. 18, pp. 1156-1157, 2005.

[73] Y. Wang, "Clinical observation of Guanxinning injection treating ischemic heart disease angina pectoris," Modern Medicine Health, vol. 21, no. 10, pp. 1280-1281, 2005.

[74] Z. B. Wang, H. J. Li, X. Y. Zhang, and S. Q. Li, "Clinical observation of Guanxinning injection in the treatment of unstable angina pectoris," Chinese Community Doctors, vol. 13, p. 163, 2011.

[75] X. F. Wu, Y. F. Wang, and J. P. Liu, "Clinical observation of guanxinning injection in the treatment of coronary heart disease and angina," China Foreign Medical Treatment, vol. 8, p. 40, 2008.

[76] Y. G. Wu, S. Zhao, X. J. Fan, and W. Q. Zhao, "Guanxinning onjection combined with Shenmai injection for unstable angina pectoris: a report of 72 cases," Journal of Anhui TCM College, vol. 30, pp. 30-33, 2011.

[77] Y. Xia, "Clinical observation of Guanxinning combined with Western medicine in the treatment of unstable angina: a report of 50 cases," Shaanxi Journal of Traditional Chinese Medicine, vol. 32, pp. 1471-1472, 2011.

[78] T. Yang and Z. X. Ma, "Clinical observation of Guanxinning combined with Western medicine in the treatment of unstable angina," Asia-Pacific Traditional Medicine, vol. 4, pp. 22-23, 2008.

[79] X. W. Ye, Y. S. Luo, and F. Gui, "Clinical observation of Low molecular weight heparin combined with Guanxinning injection in the treatment of unstable angina," Zhejiang Journal of Integrated Traditional Chinese and Western Medicine, vol. 18, pp. 424-425, 2008.

[80] H. J. Yu and W. F. Wang, "Clinical observation of Guanxinning injection combined with nitroglycerin in the treatment of angina pectoris: a report of 40 cases," Nei Mongol Journal of Traditional Chinese Medicine, vol. 6, pp. 39-40, 2009.
[81] L. Yuan, "Clinical observation of Guanxinning injection in the treatment of angina pectoris," Henan Traditional Chinese Medicine, vol. 25, pp. 71-72, 2005.

[82] L. X. Zhang, "Guanxinning injection in the treatment of angina pectoris: a report of 35 cases," Zhejiang Journal of Traditional Chinese Medicine, vol. 5, p. 229, 2005.

[83] L. X. Zhang, "Analysis study of Guanxinning injection in the treatment of unstable angina pectoris: a report of 120 cases," China Clinical Practical Medicine, vol. 4, pp. 133-134, 2010.

[84] Y. Zhang, "Clinical observation of Guanxinning combined with Ginkgo biloba in the treatment of unstable angina," Journal of Changzhi Medical College, vol. 18, pp. 179-180, 2004.

[85] Z. X. Zhang, "Nitroglycerin combined with Guanxinning injection in the treatment of unstable angina," Zhejiang Journal of Integrated Traditional Chinese and Western Medicine, vol. 14, p. 156, 2004.

[86] F. L. Zhao, X. B. Lu, C. J. Gong, P. J. Wang, L. M. Huang, and K. Guo, "Clinical observation of Guanxinning injection combined with Xueshuantong in the treatment of unstable angina," Journal of Practical Traditional Chinese Medicine, vol. 26, pp. 375-377, 2010.

[87] P. T. Zhao and Y. An, "Clinical observation of Guanxinning Injection combined with simvastatin in the treatment of unstable angina pectoris," Contemporary Medicine, vol. 141, pp. 105$106,2008$.

[88] Y. J. Zhao, "Clinical observation of Guanxinning in the treatment of angina: a report of 43 cases," Chinese Journal of Integrated Traditional and Western Medicine in Intensive and Critical Care, vol. 8, pp. 238-239, 2010.

[89] T. H. Zhong, W. Li, Y. D. Liang et al., "Clinical observation of Guanxinning in the treatment of angina: a report of 35 cases," Chinese Medicine Modern Distance Education of China, vol. 5, pp. 20-21, 2007.

[90] L. Zhu, "Integrated traditional and Western treatment of unstable angina: a report of 80 cases," Forum on Traditional Chinese Medicine, vol. 20, p. 42, 2005.

[91] L. Wang, G. B. Zhou, P. Liu et al., "Dissection of mechanisms of Chinese medicinal formula Realgar-Indigo naturalis as an effective treatment for promyelocytic leukemia," Proceedings of the National Academy of Sciences of the United States of America, vol. 105, no. 12, pp. 4826-4831, 2008.

[92] D. W. Zhang, J. G. Liu, J. T. Feng et al., "Effects of effective components compatibility of aqueous extracts of Salviae Miltiorrhizae and Rhizoma Chuanxiong on rat myocardial ischemia/reperfusion injury," Zhongguo Wei Zhong Bing Ji Jiu Yi Xue, vol. 22, no. 2, pp. 109-112, 2010.

[93] X. J. Li and H. Y. Zhang, "Synergy in natural medicines: implications for drug discovery," Trends in Pharmacological Sciences, vol. 29, no. 7, pp. 331-332, 2008.

[94] D. Wang and A. Bakhai, Clinical Trials: A Practical Guide to Design, Analysis, and Reporting, Remedica, London, UK, 2006.

[95] M. Gheorghiade and R. O. Bonow, "Chronic heart failure in the United States: a manifestation of coronary artery disease," Circulation, vol. 97, no. 3, pp. 282-289, 1998.

[96] J. L. Tang, S. Zhan, and E. Ernst, "Review of randomized controlled trials of traditional Chinese medicine," British Medical Journal, vol. 319, no. 7203, pp. 160-161, 1999.

[97] J. Liu, L. L. Kjaergard, and C. Gluud, "Misuse of randomization: a review of Chinese randomized trials of herbal medicines for chronic hepatitis B," American Journal of Chinese Medicine, vol. 30, no. 1, pp. 173-176, 2002. 
[98] X. Guo, X. Chen, L. Li et al., "LC-MS determination and pharmacokinetic study of six phenolic components in rat plasma after taking traditional Chinese medicinal-preparation: guanxinning lyophilized powder for injection," Journal of Chromatography B, vol. 873, no. 1, pp. 51-58, 2008.

[99] S. M. Yang and G. P. Deng, "The evolvement in clinical use of Perhexiline injection," Modern Hospital, vol. 7, no. 11, pp. 68-70, 2007. 


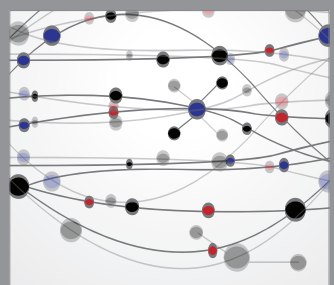

The Scientific World Journal
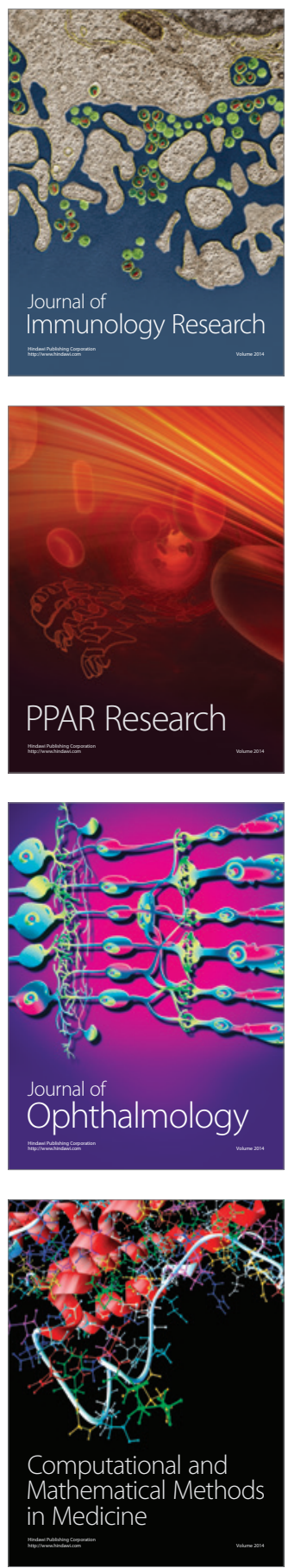

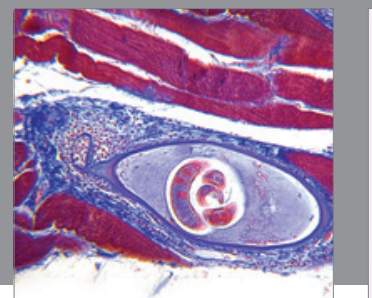

Gastroenterology

Research and Practice
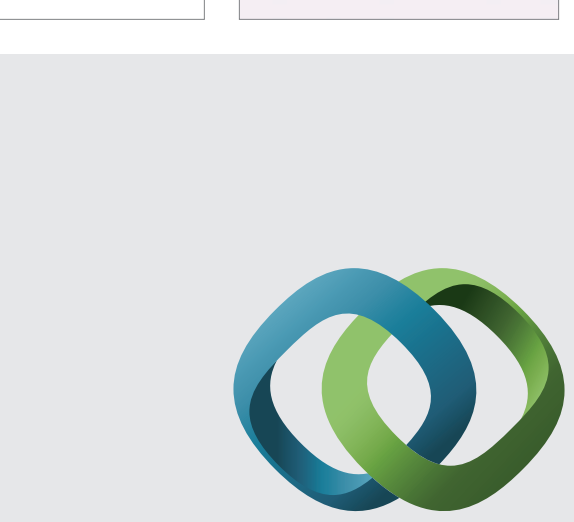

\section{Hindawi}

Submit your manuscripts at

http://www.hindawi.com
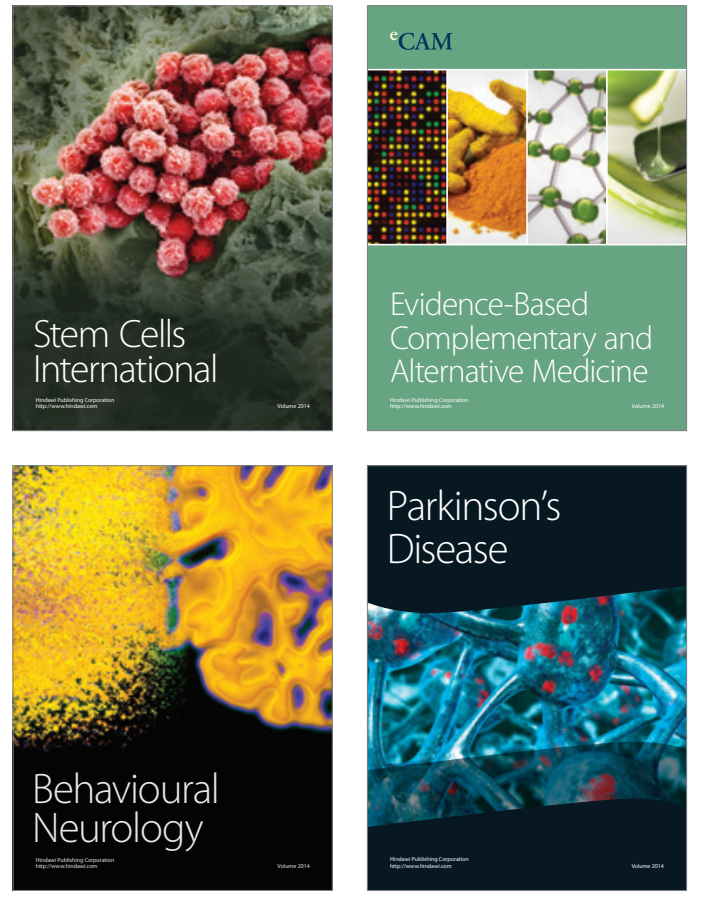
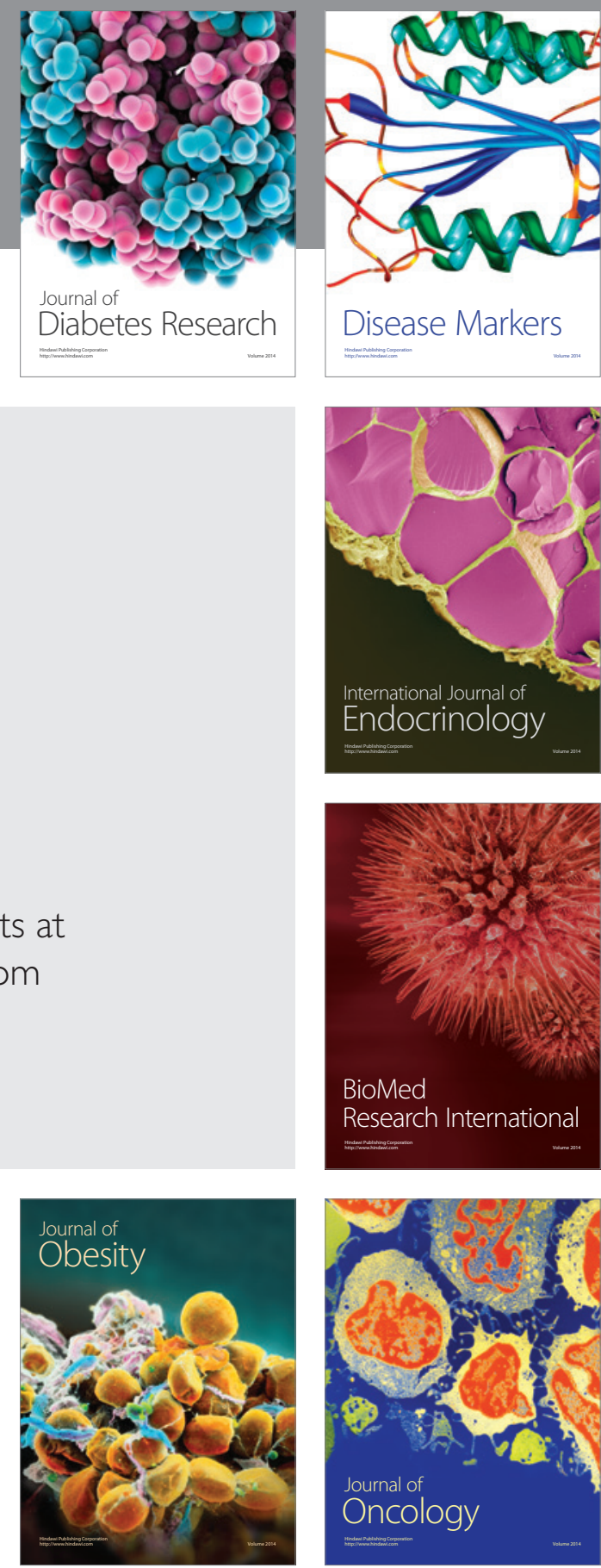

Disease Markers
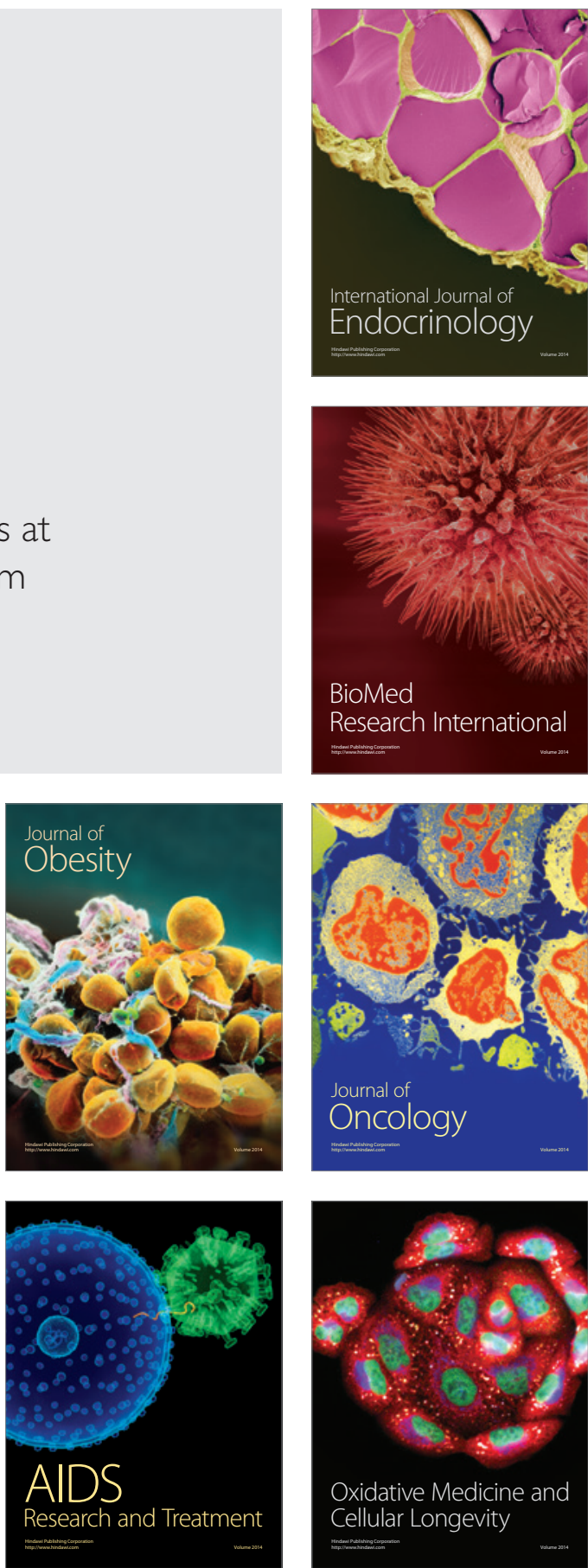